\title{
SCHRÖDINGER FLOW FOR MAPS INTO KÄHLER MANIFOLDS*
}

\author{
PETER Y. H. PANG ${ }^{\dagger}$, HONG-YU WANG ${ }^{\ddagger}$, AND YOU-DE WANG $§$
}

\begin{abstract}
In this paper, we show that there exists a global Schrödinger flow for a map from the unit circle $S^{1}$ into a Kähler manifold with constant holomorphic sectional curvature. Moreover, the Schrödinger flow obeys a conservative law. We also discuss the Schrödinger flow from a compact Riemann surface into a compact Kähler manifold and prove that the Cauchy problem admits a smooth local solution.
\end{abstract}

1. Introduction. Recently, in [DW], Ding and Wang considered Schrödinger flows for maps into symplectic manifolds. Let $(N, \omega, J, h)$ (sometimes denoted just by $N,(N, J)$ or $(N, J, h))$ be a symplectic manifold equipped with a symplectic form $\omega$, an almost complex structure $J$, and the Riemannian metric $h(\cdot, \cdot)=\omega(\cdot, J \cdot)$. Then, given a map $u_{0}$ from a Riemannian manifold $(M, g)$ into $(N, J)$, the Schrödinger flow $u(\cdot, t): M \longrightarrow N$ for $u_{0}$ is defined by the Cauchy problem

$$
\left\{\begin{array}{l}
\frac{\partial u}{\partial t}=J(u) \tau(u), \\
u(x, 0)=u_{0}(x),
\end{array}\right.
$$

where $\tau(u)$ is the tension field of $u$. In local coordinates,

$$
\tau^{\alpha}(u)=\Delta u^{\alpha}+g^{i j} \Gamma_{\beta \gamma}^{\alpha}(u) \frac{\partial u^{\beta}}{\partial x^{i}} \frac{\partial u^{\gamma}}{\partial x^{j}}
$$

where $\Delta$ is the Laplace-Beltrami operator on $M$ with respect to the metric $g$ and $\Gamma_{\beta \gamma}^{\alpha}$ are the Christoffel symbols of the target manifold $N$.

For the case where $M=S^{1}$ and $(N, J, h)$ is a Kähler manifold, Ding and Wang [DW] discussed existence and uniqueness for (1.1). They proved that it admits a unique local smooth solution if $u_{0}$ is smooth. Further, if $(N, J, h)$ is a compact Kähler manifold with constant sectional curvature $K$, the solution is in fact global and smooth. This follows from the conservative law

$$
\frac{d}{d t}\left\{\int_{S^{1}}|\tau(u)|^{2} d S-\frac{K}{4} \int_{S^{1}}|\nabla u|^{4} d S\right\}=0 .
$$

We point out that if $N$ is a compact Kähler manifold with constant sectional curvature, then it is either a closed surface or a flat complex torus of higher dimension.

We recall that the Heisenberg spin chain system (also called ferromagnetic spin chain system) is given by

$$
\frac{\partial u}{\partial t}=u \times \Delta u
$$

*Received September 19, 2000; accepted for publication October 2, 2000. Partially supported by National University of Singapore Academic Research Fund Grant RP3982718.

${ }^{\dagger}$ Department of Mathematics, National University of Singapore, 2 Science Drive 2, Republic of Singapore 117543 (matpyh@nus.edu.sg).

$\ddagger$ Department of Mathematics, National University of Singapore, 2 Science Drive 2, Republic of Singapore 117543. Current Address: Department of Mathematics, Yangzhou University, Yangzhou, Jiangsu 225009, People's Republic of China (hywang@yzu.edu.cn).

$\S$ Institute of Mathematics, Academy of Mathematics and Systems Sciences, Chinese Academy of Sciences, Beijing, People's Republic of China (wyd@math03.math.ac.cn). This author is also supported by the Natural Science Foundation of China. Work done while the author was visiting the Department of Mathematics, National University of Singapore; hospitality extended gratefully acknowledged. 
where $u$ takes values in $S^{2} \subset R^{3}$ and $\times$ denotes the cross product in $R^{3}$. Zhou, Guo and Tan in [ZGT] showed that for smooth Cauchy data there exists a unique smooth solution for the Cauchy problem of the Heisenberg spin chain system from $S^{1}$ into $S^{2}$. In [W] and [W1] Wang proved that there is a global weak solution for the Cauchy problem of the Heisenberg spin chain system (with or without external magnetic field) from any closed manifold into $S^{2}$. We note that

(i) $J(u)=u \times: T_{u} S^{2} \longrightarrow T_{u} S^{2}$ is the standard complex structure on $S^{2}$, and

(ii) the tension field of the map $\mathrm{u}$ into $S^{2}$ is given by $\tau(u)=\Delta u+|\nabla u|^{2} u$. Hence,

$$
u \times \Delta u=J(u) \tau(u)
$$

i.e., the Heisenberg spin chain system is in fact the Schrödinger flow into $S^{2}$. The equivalence of the Heisenberg spin chain system to the Schrödinger flow can also be seen by applying the stereographic projection $S^{2} \longrightarrow C \cup\{\infty\}$. Using the above transformation to define $z$ as the complex-valued representation for $u$, the Heisenberg spin chain system can be written as

$$
\frac{\partial z}{\partial t}=i\left(\Delta z-\frac{2 \bar{z}}{1+|z|^{2}}(\nabla z)^{2}\right) .
$$

Also recall the nonlinear Schrödinger equation

$$
i \psi_{t}+\psi_{x x}+2 \kappa|\psi|^{2} \psi=0
$$

where $\kappa \neq 0$ is a constant. This equation, which has many applications such as nonlinear optics, has been widely studied, see e.g. [ZS]. In particular, the lattice nonlinear Schrödinger equations with $\kappa= \pm 1$ can be written respectively as Hamiltonian equations on $S^{2}$ and the Lobachevskian plane, and thus represent respectively $S U(2)$ and $S U(1,1)$ magnetic models, see [FT] for details. Zakharov and Takhtajan in [ZT] and Lakshmanan in $[\mathrm{L}]$ pointed out that the Heisenberg spin chain system is gauge equivalent to the nonlinear Schrödinger equation with $\kappa=1$, thus establishing a deep relation between these two integrable systems.

Recently, Chang, Shatah and Uhlenbeck [CSU] employed a generalized Hasimoto transformation to show that the nonlinear Schrödinger equations with $\kappa= \pm 1$ are equivalent to the Schrödinger flows for maps from $R^{1}$ into $S^{2}$ and $H^{2}$ respectively. Moreover, they considered the following Cauchy problem for the Schödinger flow into a compact Riemannian surface:

$$
\left\{\begin{array}{l}
\frac{\partial u}{\partial t}=J(u) \tau(u), \quad x \in R^{m}, \quad m=1,2 \\
u(x, 0)=u_{0}(x)
\end{array}\right.
$$

By the Hasimoto transformation they showed that for $m=1$ and smooth Cauchy data $u_{0}(x)$, the equation (1.2) admits a unique global smooth solution. For $m=2$, they considered radially symmetric maps, and equivariant maps when the target surface has $S^{1}$ symmetry, and proved global existence and uniqueness in the small energy case (see [CSU] for details). Independently, Ding in [D] pointed out that the nonlinear Schrödinger equation with $\kappa=-1$ is gauge equivalent to the the Schrödinger flow from $R^{1}$ into $H^{2}$. He approached that problem by an appropriate choice of Lax pair for the the nonlinear Schrödinger flow of maps from $R^{1}$ into $H^{2}$ according to 
the dynamical behavior of an auxiliary linear problem for the nonlinear Schrödinger equation with $\kappa=-1$ (see [D] for details).

If $(N, J, h)$ is a complete Kähler manifold, it is well-known that we can always choose local complex coordinates in $N$ so that the operation of $J$ is just multiplication by $i=\sqrt{-1}$. Then the Schrödinger flow from $(M, g)$ into $(N, J, h)$ with small Cauchy data can be written as

$$
\left\{\begin{array}{l}
\frac{\partial u}{\partial t}=i[\Delta u+A(u, \bar{u})(d u, d \bar{u})] \\
u(\cdot, 0)=u_{0}: M \longrightarrow C^{n}
\end{array}\right.
$$

Such Cauchy problems have been studied by Kenig et al in [KPV] and Hayashi and Hirata in $[\mathrm{HH}]$, when a special form of the nonlinear term $A$ is assumed. For $M=$ $R^{m}$ and $u_{0}$ having small size restriction, they proved short time and even global existence and uniqueness using Fourier transform and methods of harmonic analysis. More recently, in [KPV1] Kenig, Ponce and Vega considered the Cauchy problem for nonlinear Schrödinger equation of the form

$$
\left\{\begin{array}{l}
\partial_{t} u=i \mathcal{L} u+P\left(u, \nabla_{x} u, \bar{u}, \nabla_{x} \bar{u}\right), \quad t \in R, \quad x \in R^{n} \\
u(\cdot, 0)=u_{0}: R^{n} \longrightarrow C,
\end{array}\right.
$$

where $u=u(x, t)$ is a complex valued function, $\nabla_{x} u=\left(\partial_{x_{1}} u, \cdots, \partial_{x_{n}} u\right), \mathcal{L}$ is a non-degenerate constant-coefficient second order operator

$$
\mathcal{L}=\sum_{j \leq k} \partial_{x_{j}}^{2}-\sum_{j>k} \partial_{x_{j}}^{2}, \quad \text { for some } k \in\{1, \cdots, n\},
$$

and $P: C^{2 n+2} \longrightarrow C$ is a polynomial having no constant or linear terms, i.e.,

$$
P(z)=P\left(z_{1}, \cdots, z_{2 n+2}\right)=\sum_{l_{0} \leq|\alpha| \leq d} a_{\alpha} z^{\alpha}, \quad l_{0} \geq 2 .
$$

They established that the above Cauchy problem is locally well-posed in appropriate Sobolev spaces without any size restriction on the data in any dimension (see [KPV1] and reference therein for more details). We note that the case $\mathcal{L}=\Delta$ had been considered previously by Chihara $[\mathrm{Ch}]$, and refer the readers to $[\mathrm{B}]$ and $[\mathrm{Cz}]$ for the semilinear case $P=P(u, \bar{u})$. In particular, Bourgain considered the initial value problem for the periodic nonlinear Schrödinger equation over $T^{n} \equiv R^{n} / Z^{n}$ as follows

$$
i \partial_{t} u+\Delta u+u|u|^{p-2}=0 \quad(p \geq 3)
$$

with initial data

$$
u(x, 0)=\phi(x)
$$

In [B] Bourgain obtained local and global results on the well-posedness of the above initial value problem in one and several space dimensions for initial data $\phi \in H^{\alpha}\left(T^{n}\right)$ for essentially optimal $\alpha$.

In this paper, we consider the Schödinger flow from $S^{1}$ into a complete Kähler manifold with constant holomorphic sectional curvature. Examples of such manifolds include complex projective space $C P^{n}$ with the Fubini-Study metric, and complex hyperbolic space $C H^{n}$ with the Bergmann metric and its compact quotients by isometric 
cocompact lattice groups. We will derive a new conservative law for the solutions of the Cauchy problem.

Theorem 1. Let $M=S^{1}$ and $(N, J, h)$ be a compact Kähler manifold with constant holomorphic sectional curvature $K$. If $u$ is a smooth solution of the Schrödinger flow from $S^{1}$ into $N$, then $u$ obeys the following conservative law:

$$
\frac{d}{d t}\left\{\int_{S^{1}}|\tau(u)|^{2} d S-\frac{K}{4} \int_{S^{1}}|\nabla u|^{4} d S\right\} \equiv 0 .
$$

Using the above conservative law, we can prove the following two theorems:

TheOREM 2. Let $(N, J, h)$ be a compact Kähler manifold with constant holomorphic sectional curvature and let $u_{0}: S^{1} \longrightarrow N$ be a smooth map. Then, the Cauchy problem for the Schrödinger flow from $S^{1}$ into $N$ with the Cauchy data $u_{0}$ admits a unique global smooth solution.

Theorem 3. The Cauchy problem for the Schrödinger flow from $S^{1}$ into complex hyperbolic space $\mathrm{CH}^{n}$ (with the Bergmann metric) with smooth Cauchy data $u_{0}$ admits a unique global smooth solution.

Finally, in this paper, we consider 2-dimensional Schrödinger flows, i.e., where $M$ is a Riemann surface. We have the following local existence and uniqueness result:

TheOREM 4. Let $M$ be a closed Riemann surface and $N$ a compact Kähler manifold with nonpositive sectional curvature. Let $u_{0}$ be a smooth map from $M$ into $N$. Then, the Cauchy problem for the Schrödinger flow from $M$ into $N$ with Cauchy data $u_{0}$ admits a unique local smooth solution.

The key step for proving the local existence and uniqueness is to establish a relation between the Sobolev norms of $d u$ and the tension field $\tau(u)$, and then adopt a Landau-Lifshitz type equation to approximate the Schrödinger flow equation. To contrast our results with those obtained in [KPV1], we remark that the term containing the first order derivatives in the Schrödinger flow equation cannot be expressed as a polynomial in general. For instance, the Schrödinger flow into the Poincaré disk, which is equivalent to the anisotropic ferromagnetic system, is given by the equation

$$
\begin{aligned}
\frac{\partial z}{\partial t} & =i\left(\Delta z+\frac{2 \bar{z}}{1-|z|^{2}}(\nabla z)^{2}\right) \\
& =i\left\{\Delta z+2 \sum_{j=0}^{\infty}|z|^{2 j} \bar{z}(\nabla z)^{2}\right\}
\end{aligned}
$$

where $z(x, t) \in\{z \in C:|z|<1\}$. Thus, the Fourier analysis methods of [KPV1] do not seem to be applicable. We also note that our method can be employed to prove the local existence of the inhomogeneous Schrödinger flow from a compact manifold into a compact Kähler manifold. This has been treated in a separate paper [PWW].

This paper is organized as follows: In Section 2, we recall some facts and notations in differential geometry, and establish some relations on the Sobolev norms. In Section 3 , we prove the conservative law (Theorem 1) and use it to prove global existence and uniqueness (Theorems 2 and 3). Section 4 is devoted to proving Theorem 4 .

A note on notation. We shall use the symbol $C$ generically to denote certain scalar-valued terms in the estimates to be derived in the remainder of the paper. We will, however, normally specify the objects/quantities on which these terms depend, by 
means of arguments to $C$. For example, the symbol $C(M, N)$ shall denote a constant depending only on the manifolds $M$ and $N$, whereas the symbol $C\left(\|\tau(u)\|_{L^{2}}, E(u)\right)$ shall denote a smooth scalar-valued function depending on the quantities $\|\tau(u)\|_{L^{2}}$ and $E(u)$. Thus, the latter $C$ is not necessarily a constant but may vary with $u$. Also, unless otherwise specified, $C$ shall be assumed to depend on its arguments smoothly.

Acknowledgement. We would like to thank Wei-Yue Ding for his valuable comments and suggestions, and especially for his interest in the conservative law.

2. Some Geometric and Analytic Preliminaries. Let $\pi: E \longrightarrow M$ be a Riemannian vector bundle over a Riemannian manifold $M$. Then, for $p=1,2, \cdots$, $\operatorname{dim}(M)$, we have the bundles $\Lambda^{p} T^{*} M \otimes E \longrightarrow M$ over $M$ whose smooth sections constitute the sets $\Gamma\left(\Lambda^{p} T^{*} M \otimes E\right)$. On $\Lambda^{p} T^{*} M \otimes E$, we have the induced metric

$$
\left\langle s_{1}, s_{2}\right\rangle=\sum_{i_{1}<i_{2}<\cdots<i_{p}}\left\langle s_{1}\left(e_{i_{1}}, \cdots, e_{i_{p}}\right), s_{2}\left(e_{i_{1}}, \cdots, e_{i_{p}}\right)\right\rangle_{E},
$$

where $\left\{e_{i}\right\}$ is a local orthonormal frame of $T M$. This induces an inner product on $\Gamma\left(\Lambda^{p} T^{*} M \otimes E\right)$ as follows:

$$
\left(s_{1}, s_{2}\right)=\int_{M}\left\langle s_{1}, s_{2}\right\rangle d M
$$

The space $L^{2}\left(M, \Lambda^{p} T^{*} M \otimes E\right)$ is the completion of $\Gamma\left(\Lambda^{p} T^{*} M \otimes E\right)$ with respect to the above inner product $(\cdot, \cdot)$. Analogously, we may also define the Sobolev spaces $H^{k, r}\left(M, \Lambda^{p} T^{*} M \otimes E\right)$ (see Appendix in [DK] for details) as follows: Let $\nabla$ be the covariant differential induced by the metric on $\Lambda^{p} T^{*} M \otimes E$, then the bundle-valued Sobolev spaces $H^{k, r}\left(M, \Lambda^{p} T^{*} M \otimes E\right)$ are defined by taking completions with respect to the norms

$$
\|s\|_{k, r}=\left(\sum_{i=0}^{k} \int_{M}\left|\nabla^{i} s\right|^{r} d M\right)^{\frac{1}{r}}
$$

where

$$
\left|\nabla^{i} s\right|=\langle\underbrace{\nabla \cdots \nabla}_{i \text { times }} s, \underbrace{\nabla \cdots \nabla}_{i \text { times }} s\rangle^{\frac{1}{2}} .
$$

In the case where $M$ is a compact Riemannian manifold, we recall two standard results on Sobolev spaces, namely, the Rellich theorem and Sobolev imbedding theorem. Also, in the rest of the paper, we shall use the short-hand notation $\nabla_{i}$ for $\nabla_{e_{i}}$ where $\left\{e_{i}\right\}$ is a local orthonormal frame of $T M$.

On the other hand, it is well-known that a compact Riemannian manifold $N$ can be isometrically embedded into a Euclidean space $R^{d}$ for some positive integer $d>\operatorname{dim}(N)$. Hence, one can define the Sobolev spaces $W^{k, p}(M, N)=\{g: g \in$ $W^{k, p}\left(M, R^{d}\right), g(x) \in N$ for a.e. $\left.x \in M\right\}$. These spaces are sometimes called Sobolev spaces of maps. Our main task in this section is to establish some relations between the norms of the Sobolev spaces of maps and the bundle-valued Sobolev spaces.

In terms of the covariant exterior differential operator $d: \Gamma\left(\Lambda^{p} T^{*} M \otimes E\right) \longrightarrow$ $\Gamma\left(\Lambda^{p+1} T^{*} M \otimes E\right)$ defined by

$$
d s\left(X_{0}, \cdots, X_{p}\right)=(-1)^{k}\left(\nabla_{X_{k}} s\right)\left(X_{0}, \cdots, \hat{X}_{k}, \cdots, X_{p}\right)
$$


and its formal adjoint $\delta: \Gamma\left(\Lambda^{p} T^{*} M \otimes E\right) \longrightarrow \Gamma\left(\Lambda^{p-1} T^{*} M \otimes E\right)$ defined by

$$
\delta s\left(X_{1}, \cdots, X_{p-1}\right)=-\left(\nabla_{i} s\right)\left(e_{i}, X_{1}, \cdots, X_{p-1}\right),
$$

where $X_{0}, X_{1}, \cdots, X_{p} \in \Gamma(T M)$, the Hodge-Laplace operator is given by

$$
\Delta_{H}=d \delta+\delta d
$$

It is well-known that $\Delta_{H}$ is a self-adjoint, positive semi-definite elliptic operator, and the following Weitzenböck formula holds:

Proposition 2.1. For any $s \in \Gamma\left(\Lambda^{p} T^{*} M \otimes E\right)$,

$$
\Delta_{H} s=-\Delta s+S,
$$

where $\Delta=-\nabla^{*} \nabla$ is the Laplace-Beltrami operator and

$$
S\left(X_{1}, \cdots, X_{p}\right)=(-1)^{k}\left(R\left(e_{i}, X_{k}\right) s\right)\left(e_{i}, X_{1}, \cdots, \hat{X}_{k}, \cdots, X_{p}\right) .
$$

Here, $R(\cdot, \cdot)$ is the curvature operator associated to the induced connection on the vector bundle $\Lambda^{p} T^{*} M \otimes E$.

Let $f: M \longrightarrow N$ be a smooth map and regard $d f \in \Gamma\left(T^{*} M \otimes f^{*} T N\right)$. We note that

$$
\begin{aligned}
d(d f)\left(X_{1}, X_{2}\right) & =\left(\nabla_{X_{1}} d f\right)\left(X_{2}\right)-\left(\nabla_{X_{2}} d f\right)\left(X_{1}\right) \\
& =A_{X_{1} X_{2}}(f)-A_{X_{2} X_{1}}(f) \\
& =0
\end{aligned}
$$

where $A_{X_{1} X_{2}}(f)$ is the second fundamental form of $f$ which is symmetric. On the other hand, we have (see [EL])

$$
\delta(d f)=-\left(\nabla_{i} d f\right)\left(e_{i}\right)=- \text { trace }_{g} \nabla d u=-\tau(f) .
$$

Hence, $\tau(f) \equiv 0$ is equivalent to the harmonicity of the $\Gamma\left(f^{*} T N\right)$-valued 1-form $d f$. In other words, $f$ is a harmonic map if and only if $\Delta_{H}(d f)=0$.

The energy of the map $f$ is defined by

$$
E(f)=\frac{1}{2} \int_{M}|\nabla f|^{2} d M
$$

To describe the tension field of $f$, one usually adopts local frames on $M$ and $N$. Let $\left\{e_{i}\right\}$ be a local orthonormal frame on $M$ and $\left\{e_{i}^{*}\right\}$ its dual frame. Let $\left\{\bar{e}_{\alpha}\right\}$ be a local orthonormal frame on $N$. Then, with respect to the above frames, $d f=f_{i}^{\alpha} e_{i}^{*} \otimes \bar{e}_{\alpha}$, and $\tau(f)=\nabla_{i} f_{i}$ where $f_{i}=f_{i}^{\alpha} \bar{e}_{\alpha}=f_{*} e_{i}$.

From the Weitzenböck formula we can easily derive:

Proposition 2.2. Let $M$ be a closed Riemannian manifold and $N$ a complete Riemannian manifold with nonpositive sectional curvature. For a smooth map $u$ : $M \longrightarrow N$, there exists a constant $C(M)$ such that

$$
\int_{M}|\nabla d u|^{2} d M \leq \int_{M}|\tau(u)|^{2} d M+C(M) E(u) .
$$


In particular, if $M$ has nonnegative Ricci curvature, then

$$
\int_{M}|\nabla d u|^{2} d M \leq \int_{M}|\tau(u)|^{2} d M
$$

Proof. We note that

$$
\begin{aligned}
S(X) & =-\left(R\left(e_{i}, X\right) d f\right) e_{i} \\
& =-K^{N}\left(f_{*} e_{i}, f_{*} X\right) f_{*} e_{i}+f_{*} R i c^{M}(X),
\end{aligned}
$$

where $K^{N}(\cdot, \cdot)$ is the sectional curvature operator of $N, \operatorname{Ric}^{M}(\cdot)$ is the Ricci curvature operator of $M$, and $f_{*}$ is the tangent map from $T M$ to $T N$. Thus, by Proposition 2.1 with $p=1$ and $s=d f$,

$$
\begin{aligned}
\Delta e(f)= & \langle\Delta(d f), d f\rangle+|A(f)|^{2} \\
= & -\left\langle\Delta_{H}(d f), d f\right\rangle+|\nabla d f|^{2}+\left\langle f_{*} \operatorname{Ric}^{M}\left(e_{i}\right), f_{*} e_{i}\right\rangle \\
& -\left\langle K^{N}\left(f_{*} e_{i}, f_{*} e_{j}\right) f_{*} e_{i}, f_{*} e_{j}\right\rangle .
\end{aligned}
$$

Since the sectional curvature of $N$ is nonpositive, i.e., $\left\langle K^{N}\left(f_{*} e_{i}, f_{*} e_{j}\right) f_{*} e_{i}, f_{*} e_{j}\right\rangle \leq 0$, we get

$$
\Delta e(f) \geq-\left\langle\Delta_{H}(d f), d f\right\rangle+|\nabla d f|^{2}+\left\langle f_{*} \operatorname{Ric}^{M}\left(e_{i}\right), f_{*} e_{i}\right\rangle
$$

The result now follows by integrating the two sides of (2.3) over $M$ and noting $\delta(d u)=$ $-\tau(u)$.

In the following two results, $(N, h)$ is regarded as an isometrically embedded submanifold in the Euclidean space $R^{d}$. For convenience, we denote $\|\cdot\|_{C^{0}\left(M, R^{d}\right)}$ and $\|\cdot\|_{W^{k, p}\left(M, R^{d}\right)}$ by $\|\cdot\|_{C^{0}}$ and $\|\cdot\|_{W^{k, p}}$ respectively. A word of caution on notations: we use $\nabla$ to denote both the covariant derivative induced by the Riemannian metric on $M$ and the covariant differential on $\otimes^{p} T^{*} M \otimes u^{*} T N$ induced by the Riemannian metrics on $M$ and $N$, with the distinction given by the context. In the former case, for any smooth map $u: M \longrightarrow N$, its tangent map $d u$ is equal to $\nabla u$; both notations will be used.

Proposition 2.3. Let $M$ be a closed Riemann surface and $N$ a compact Riemannian manifold with nonpositive sectional curvature. For a smooth map $u: M \longrightarrow N$, there exists a constant $C(M, N)$ such that

$$
\|d u\|_{W^{2,2}} \leq C(M, N)\left\{(E(u)+\sqrt{E(u)}+1)\left(\|\nabla \tau(u)\|_{L^{2}}^{\frac{3}{2}}+\|\nabla \tau(u)\|_{L^{2}}\right)+C(E(u))\right\} .
$$

In particular, if $M$ has nonnegative Ricci curvature, then

$$
\|d u\|_{W^{2,2}} \leq C(M, N)\left\{(E(u)+\sqrt{E(u)})\|\nabla \tau(u)\|_{L^{2}}^{\frac{3}{2}}+\|\nabla \tau(u)\|_{L^{2}}+C(E(u))\right\} .
$$

Proof. We first discuss the case where $M$ has nonnegative Ricci curvature. From the identity

$$
\int_{M}\langle\tau(u), \tau(u)\rangle d M=-\int_{M}\left\langle\nabla_{i} \tau(u), u_{*} e_{i}\right\rangle d M
$$


we get

$$
\int_{M}\langle\tau(u), \tau(u)\rangle d M \leq\left(\int_{M}|\nabla \tau(u)|^{2} d M\right)^{\frac{1}{2}}\left(\int_{M}|d u|^{2} d M\right)^{\frac{1}{2}} .
$$

As $\tau(u)$ is a semilinear elliptic operator with a square term in the first order derivatives, by standard elliptic theory, for any $p>1$, we have

$$
\|d u\|_{W^{1, p}} \leq C(M, N)\left\{\|\tau(u)\|_{L^{p}}+\|d u\|_{L^{2 p}}^{2}\right\} .
$$

By the Kato inequality and Sobolev imbedding theorem, we have

$$
\begin{aligned}
\|\tau(u)\|_{L^{p}} & \leq C(M)\left\{\|\nabla \mid \tau(u)\|_{L^{2}}+\|\tau(u)\|_{L^{2}}\right\} \\
& \leq C(M)\left\{\|\nabla \tau(u)\|_{L^{2}}+\|\tau(u)\|_{L^{2}}\right\}
\end{aligned}
$$

and

$$
\begin{aligned}
\|d u\|_{L^{2 p}}^{2} & \leq C(M)\left\{\|\nabla \mid d u\|_{L^{2}}^{2}+\|d u\|_{L^{2}}^{2}\right\} \\
& \leq C(M)\left\{\|\nabla d u\|_{L^{2}}^{2}+\|d u\|_{L^{2}}^{2}\right\} .
\end{aligned}
$$

Combining (2.4)-(2.7), and using Proposition 2.2, we obtain

$$
\begin{aligned}
\|d u\|_{W^{1, p}} & \leq C(M, N)\left\{\|\nabla \tau(u)\|_{L^{2}}+\|\tau(u)\|_{L^{2}}+\|\nabla d u\|_{L^{2}}^{2}+\|d u\|_{L^{2}}^{2}\right\} \\
& \leq C(M, N)\left\{\|\nabla \tau(u)\|_{L^{2}}+\|\tau(u)\|_{L^{2}}^{2}+\|\tau(u)\|_{L^{2}}+\|d u\|_{L^{2}}^{2}\right\} \\
& \leq C(M, N)\left\{\left(1+\|d u\|_{L^{2}}\right)\|\nabla \tau(u)\|_{L^{2}}+\|\tau(u)\|_{L^{2}}+\|d u\|_{L^{2}}^{2}\right\}
\end{aligned}
$$

Hence, for $p>2$, the last inequality in (2.8) and the Sobolev imbedding theorem for functions imply that

$$
\|d u\|_{C^{0}} \leq C(M, N)\left\{\left(1+\|d u\|_{L^{2}}\right)\|\nabla \tau(u)\|_{L^{2}}+\|\tau(u)\|_{L^{2}}+\|d u\|_{L^{2}}^{2}\right\} .
$$

From (2.9) and (2.4) we deduce that

$$
\begin{aligned}
\|d u\|_{C^{0}} & \leq C(M, N)\left\{\left(1+\|d u\|_{L^{2}}\right)\|\nabla \tau(u)\|_{L^{2}}+\|d u\|_{L^{2}}+\|d u\|_{L^{2}}^{2}\right\} \\
& =C(M, N)\left\{(1+\sqrt{E(u)})\|\nabla \tau(u)\|_{L^{2}}+\sqrt{E(u)}+E(u)\right\}
\end{aligned}
$$

Similarly, by standard elliptic regularity theory,

$$
\begin{aligned}
\|d u\|_{W^{2,2}} & \leq C(M, N)\left\{\|\nabla \tau(u)\|_{L^{2}}+\|d u\|_{L^{2}}+\left[\int_{M}|\nabla d u|^{2}|d u|^{2} d M\right]^{\frac{1}{2}}\right\} \\
& \leq C(M, N)\left\{\|\nabla \tau(u)\|_{L^{2}}+\|d u\|_{L^{2}}+\|\nabla d u\|_{L^{2}}\|d u\|_{C^{0}}\right\} \\
& \leq C(M, N)\left\{\|\nabla \tau(u)\|_{L^{2}}+\|d u\|_{L^{2}}+\|\tau(u)\|_{L^{2}}\|d u\|_{C^{0}}\right\} .
\end{aligned}
$$

From (2.4), (2.10) and the preceding inequality we conclude that

$(2.11)\|d u\|_{W^{2,2}} \leq C(M, N)\left\{(E(u)+\sqrt{E(u)})\|\nabla \tau(u)\|_{L^{2}}^{\frac{3}{2}}+\|\nabla \tau(u)\|_{L^{2}}+C(E(u))\right\}$.

For the general case, a modification of the above arguments yields

$$
\begin{aligned}
\|d u\|_{W^{2,2}} \leq & C(M, N)\left\{(E(u)+\sqrt{E(u)})\|\nabla \tau(u)\|_{L^{2}}^{\frac{3}{2}}\right. \\
& \left.+(E(u)+\sqrt{E(u)}+1)\|\nabla \tau(u)\|_{L^{2}}+C(E(u))\right\}
\end{aligned}
$$


from which the desired inequality follows.

Proposition 2.4. With the same assumptions as in Proposition 2.3, for $k \geq 1$, there exist constants $C(M, N)$ such that

$\|d u\|_{W^{2 k+1,2}} \leq C(M, N)\left\|\Delta^{k} \tau(u)\right\|_{L^{2}}+C_{2 k+1}\left(\left\|\nabla \Delta^{k-1} \tau(u)\right\|_{L^{2}}, \cdots,\|\nabla \tau(u)\|_{L^{2}}, E(u)\right)$, and

$$
\|d u\|_{W^{2 k+2,2}} \leq C(M, N)\left\|\nabla \Delta^{k} \tau(u)\right\|_{L^{2}}+C_{2 k+2}\left(\left\|\Delta^{k} \tau(u)\right\|_{L^{2}}, \cdots,\|\nabla \tau(u)\|_{L^{2}}, E(u)\right) .
$$

Proof. Denoting the second fundamental form of the isometric embedding $N \subset R^{d}$ by $A_{\tilde{\beta} \tilde{\gamma}}^{\tilde{\alpha}}$, the tension field can be written as

$$
\tau^{\tilde{\alpha}}(u(x))=\Delta u^{\tilde{\alpha}}(x)+g^{i j}(x) A^{\tilde{\alpha}}\left(\frac{\partial u}{\partial x^{i}}, \frac{\partial u}{\partial x^{j}}\right) .
$$

Without loss of generality, we may assume that $M$ is a flat torus, i.e., $M \equiv T^{m}$.

We proceed by induction. For $k=1$, with respect to the natural local frame $\left\{\frac{\partial}{\partial y^{\alpha}}\right\}$ of $R^{d}$

$$
\nabla_{i} \tau(u)=\left(\nabla_{i} \tau(u)\right)^{\tilde{\alpha}} \frac{\partial}{\partial y^{\tilde{\alpha}}}
$$

By a direct computation, we have

$$
\left(\nabla_{i} \tau(u)\right)^{\tilde{\alpha}}=\frac{\partial}{\partial x^{i}}\left\{\tau^{\tilde{\alpha}}(u)\right\}+\frac{\partial u^{\tilde{\lambda}}}{\partial x^{i}} A_{\tilde{\lambda} \tilde{\mu}}^{\tilde{\alpha}}(u)\left\{\tau^{\tilde{\mu}}(u)\right\}
$$

and

$$
\begin{aligned}
(\Delta \tau(u))^{\tilde{\alpha}} & =\frac{\partial}{\partial x^{i}}\left\{\left(\nabla_{i} \tau(u)\right)^{\tilde{\alpha}}\right\}+\frac{\partial u^{\tilde{\lambda}}}{\partial x^{i}} A_{\tilde{\lambda} \tilde{\mu}}^{\tilde{\alpha}}(u)\left\{\left(\nabla_{i} \tau(u)\right)^{\tilde{\mu}}\right\} \\
& =\Delta^{2} u^{\tilde{\alpha}}+P_{1}(d u, \nabla d u)+P_{2}(d u) * \nabla^{2} d u
\end{aligned}
$$

where $P_{i}$ are polynomials with matrix (or vector) values and

$$
\begin{aligned}
P_{2}(d u) * \nabla^{2} d u & =P_{i_{1} i_{2} i_{3}}(d u) \nabla_{i_{1}} \nabla_{i_{2}} \nabla_{i_{3}} u \\
& =P_{i_{1} i_{2} i_{3}}(d u) \nabla_{i_{1}} \nabla_{i_{2}} u_{i_{3}}
\end{aligned}
$$

for $i_{1}, i_{2}, i_{3} \in\{1,2\}$ (here we have suppressed reference to the $\tilde{\alpha}$-th component). Applying the Sobolev imbedding theorem and Proposition 2.3 to (2.13), we see that

$$
\left\|P_{1}(d u, \nabla d u)\right\|_{L^{2}} \leq C\left(\|\nabla \tau(u)\|_{L^{2}}, E(u)\right),
$$

and

$$
\left\|P_{2}(d u) * \nabla^{2} d u\right\|_{L^{2}} \leq C\left(\|\nabla \tau(u)\|_{L^{2}}, E(u)\right) .
$$

Hence, by standard elliptic theory,

$$
\|d u\|_{W^{3,2}} \leq C(M, N)\|\Delta \tau(u)\|_{L^{2}}+C\left(\|\nabla \tau(u)\|_{L^{2}}, E(u)\right) .
$$


When $k \geq 1$, with respect to the natural local frame $\left\{\frac{\partial}{\partial y^{\bar{\alpha}}}\right\}$,

$$
\Delta^{k} \tau(u)=\left(\Delta^{k} \tau(u)\right)^{\tilde{\alpha}} \frac{\partial}{\partial y^{\tilde{\alpha}}} .
$$

Similarly to (2.12), we have

$$
\begin{aligned}
\nabla_{i} \Delta^{k} \tau(u) & =\frac{\partial}{\partial x^{i}}\left(\Delta^{k} \tau(u)\right)^{\tilde{\alpha}}+\frac{\partial u^{\tilde{\lambda}}}{\partial x^{i}} A_{\tilde{\lambda} \tilde{\mu}}^{\tilde{\alpha}}(u)\left\{\left(\Delta^{k} \tau(u)\right)^{\tilde{\mu}}\right\} \\
& =\Delta^{k+1} \frac{\partial u}{\partial x^{i}}+P_{1}^{\prime}\left(d u, \nabla d u, \cdots, \nabla^{2 k} d u\right)+P_{2}^{\prime}(d u) * \nabla^{2 k+1} d u
\end{aligned}
$$

where

$$
P_{2}^{\prime}(d u) * \nabla^{2 k+1} d u=P_{i_{1} i_{2} \cdots i_{2 k+2}}^{\prime}(d u) \nabla_{i_{1}} \nabla_{i_{2}} \cdots \nabla_{i_{2 k+1}} u_{i_{2 k+2}}
$$

for $i_{1}, i_{2}, \cdots, i_{2 k+2} \in\{1,2\}$. We leave the details of the induction step to the reader.

We end the section with the following proposition concerning the interpolation inequality (see $[\mathrm{Au}])$ :

Proposition 2.5. Let $E \longrightarrow M$ be a Riemannian vector bundle over a closed Riemannian manifold with $\operatorname{dim} M=m$. For $s \in \Gamma(E)$, there exists a constant $C(M)$, which does not depend on $E$, such that

$$
\|\nabla s\|_{L^{p}} \leq C(M)\|\nabla s\|_{H^{1, r}}^{a}\|s\|_{L^{q}}^{1-a},
$$

where

$$
\frac{1}{p}=\frac{1}{m}+a\left(\frac{1}{r}-\frac{2}{m}\right)+(1-a) \frac{1}{q}
$$

for all $a$ in the interval $\frac{1}{2} \leq a \leq 1$ for which $p$ is nonnegative.

Proof. We begin by establishing the interpolation inequality

$$
\|\nabla s\|_{L^{p}}^{2} \leq(\sqrt{m}+|p-2|)\|s\|_{L^{q}}\left\|\nabla^{2} s\right\|_{L^{r}},
$$

where $\frac{2}{p}=\frac{1}{q}+\frac{1}{r}$. First, suppose $p \geq 2$. Then, for $s \in \Gamma(E)$, we have

$$
\begin{aligned}
\nabla_{i}\left(|\nabla s|^{p-2}\left\langle s, \nabla_{i} s\right\rangle\right)= & |\nabla s|^{p}+|\nabla s|^{p-2}\langle s, \Delta s\rangle \\
& +(p-2)|\nabla s|^{p-4}\left\langle s, \nabla_{i} s\right\rangle\left\langle\nabla_{i} \nabla_{j} s, \nabla_{j} s\right\rangle .
\end{aligned}
$$

Integrating the above identity over $M$, we get

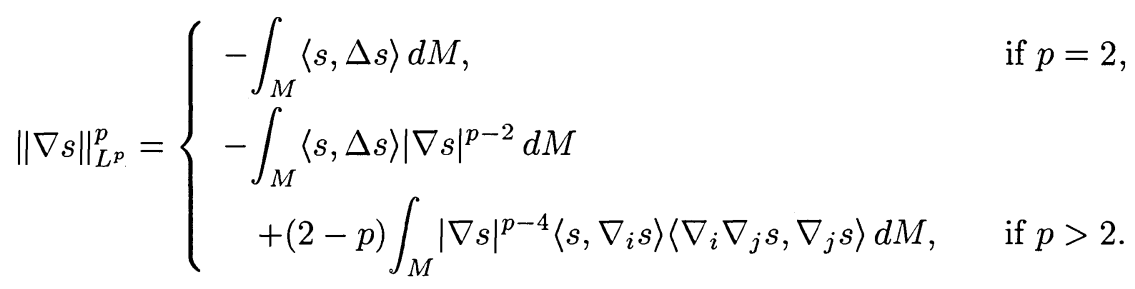

Noting that $|\Delta s|^{2} \leq m\left|\nabla^{2} s\right|^{2}$ and $\left|\left\langle s, \nabla_{i} s\right\rangle\left\langle\nabla_{i} \nabla_{j} s, \nabla_{j} s\right\rangle\right| \leq\left|\nabla^{2} s \| \nabla s\right|^{2}|s|$, we get

$$
\|\nabla s\|_{L^{p}}^{p} \leq(\sqrt{m}+|p-2|) \int_{M}\left|\nabla^{2} s \| \nabla s\right|^{p-2}|s| d M .
$$


As $\frac{2}{p}=\frac{1}{q}+\frac{1}{r},(2.17)$ now follows from the Hölder inequality.

When $1 \leq p<2$, the proof is similar, but a little more delicate (see $[\mathrm{Au}]$ ).

To complete the proof of Proposition 2.5, we need to discuss the the following two cases:

Case 1: $r<m$. The desired inequality for $a=1$ is just the Sobolev imbedding theorem, while for $a=\frac{1}{2}$, it reduces to (2.17). Hence we can deduce the required inequality for $\frac{1}{2}<a<1$. Indeed, by the Hölder inequality, for $p \in[r, q]$,

$$
\|s\|_{L^{p}} \leq\|s\|_{L^{r}}^{a}\|s\|_{L^{q}}^{1-a}
$$

where $a\left(\frac{1}{r}-\frac{1}{q}\right)=\frac{1}{p}-\frac{1}{q}$. It follows that for $\frac{1}{\rho^{\prime}}=\frac{1}{r}-\frac{1}{m}$

$$
\|\nabla s\|_{L^{p}} \leq\|\nabla s\|_{L^{\rho^{\prime}}}^{\nu}\|\nabla s\|_{L^{\rho}}^{1-\nu}
$$

where $\frac{1}{p}=\nu \frac{1}{\rho}+(1-\nu) \frac{1}{\rho^{\prime}}$. On the other hand, applying (2.17) we have

$$
\|\nabla s\|_{L^{\rho}}^{2} \leq C(m, \rho)\|s\|_{L^{q}}\left\|\nabla^{2} s\right\|_{L^{r}}
$$

where $\frac{2}{\rho}=\frac{1}{q}+\frac{1}{r}$. Hence, combining the last two inequalities and using the Sobolev imbedding theorem we obtain that

$$
\|\nabla s\|_{L^{p}} \leq C(m, \rho)\|s\|_{L^{q}}^{(1-a)}\|\nabla s\|_{H^{1, r}}^{a}
$$

where $a\left(\frac{1}{r}-\frac{1}{m}-\frac{1}{q}\right)=\frac{1}{p}-\frac{1}{m}-\frac{1}{q}$.

Case 2: $r>m$. Applying the interpolation inequality to the function $|\nabla s|$ we obtain that

$$
\|\nabla s\|_{L^{p}} \leq C(M)\left\{\|\nabla|\nabla s|\|_{L^{r}}^{2}+\|\nabla s\|_{L^{r}}^{2}\right\}^{\frac{b}{2}}\|\nabla s\|_{L^{\rho}}^{1-b}
$$

where $\frac{1}{p}=\frac{1}{\rho}+b\left(\frac{1}{r}-\frac{1}{m}-\frac{1}{\rho}\right)>0$ and $0 \leq b \leq 1$. By Kato inequality, it follows that

$$
\|\nabla s\|_{L^{p}} \leq C(M)\|\nabla s\|_{H^{1, r}}^{b}\|\nabla s\|_{L^{\rho}}^{1-b}
$$

By using (2.17) again, we have

$$
\|\nabla s\|_{L^{\rho}}^{2} \leq C(M, \rho)\|s\|_{L^{q}}\left\|\nabla^{2} s\right\|_{L^{r}}
$$

where $\frac{2}{\rho}=\frac{1}{q}+\frac{1}{r}$. Substituting $(2.20)$ into (2.19), it follows that by a direct computation that

$$
\|\nabla s\|_{L^{p}} \leq C(M)\|\nabla s\|_{H^{1, r}}^{a}\|s\|_{L^{q}}^{1-a}
$$

where

$$
\frac{1}{p}=\frac{1}{m}+a\left(\frac{1}{r}-\frac{2}{m}\right)+(1-a) \frac{1}{q} .
$$

This is the desired result. 
3. One-Dimensional Global Schrödinger Flow. In this section we discuss the global existence of smooth solutions for the Cauchy problem of the Schrödinger flow into a Kähler manifold with constant holomorphic sectional curvature. We shall refer to the following local existence result about one-dimensional Schrödinger flows from $[\mathrm{DW}]$.

Proposition 3.1. [DW] If $M=S^{1}$ and $(N, J, h)$ is a complete Kähler manifold, then the Cauchy problem (1.1) of the Schrödinger flow for maps from $S^{1}$ into $N$ with Cauchy data $u_{0} \in C^{\infty}\left(S^{1}, N\right)$ has a unique smooth solution on $S^{1} \times[0, T)$, for some $T \in(0, \infty]$. Furthermore, the energy is conserved along the solution, i.e., $E(u(x, t))=E\left(u_{0}(x)\right)$.

As remarked earlier, the conservative law (Theorem 1) is essential in proving global existence. We now give the proof of Theorem 1 which will then be exploited to prove Theorems 2 and 3 .

Proof of Theorem 1. For ease of notation, we denote $\partial u / \partial t$ and $\partial u / \partial x$ by $\dot{u}$ and $u^{\prime}$ respectively, and write $\nabla_{u_{*}\left(\frac{\partial}{\partial t}\right)}=\nabla_{t}$ and $\nabla_{u_{*}\left(\frac{\partial}{\partial x}\right)}=\nabla_{x}$. Since $u$ satisfies $\dot{u}=J(u) \tau(u)$, and $\nabla J \equiv 0$ as $(N, J, h)$ is a Kähler manifold, using the formula (see [H]) $\nabla_{t} \nabla_{x} u^{\prime}=\nabla_{x} \nabla_{x} \dot{u}+R\left(u^{\prime}, \dot{u}\right) u^{\prime}$, where $R(\cdot, \cdot) \cdot$ denotes the Riemannian curvature tensor of $N$, we have

$$
\begin{aligned}
\frac{1}{2} \frac{d}{d t} \int_{S^{1}}|\tau(u)|^{2} d S & =\frac{1}{2} \frac{d}{d t} \int_{S^{1}}|\dot{u}|^{2} d S \\
& =\int_{S^{1}}\left\langle\dot{u}, \nabla_{t}\left(J(u) \nabla_{x} u^{\prime}\right)\right\rangle d S \\
& \left.=\int_{S^{1}}\left\langle\dot{u}, \nabla_{t}(J(u)) \nabla_{x} u^{\prime}\right\rangle d S+\int_{S^{1}}\left\langle\dot{u}, J(u) \nabla_{t} \nabla_{x} u^{\prime}\right)\right\rangle d S \\
& =\int_{S^{1}}\left\langle\nabla_{x} \dot{u}, J(u) \nabla_{x} \dot{u}\right\rangle d S+\int_{S^{1}}\left\langle\dot{u}, J(u) R\left(u^{\prime}, \dot{u}\right) u^{\prime}\right\rangle d S
\end{aligned}
$$

The first integral on the right hand side of the above equality vanishes since

$$
\begin{aligned}
\left\langle\nabla_{x} \dot{u}, J(u) \nabla_{x} \dot{u}\right\rangle & =h\left(\nabla_{x} \dot{u}, J(u) \nabla_{x} \dot{u}\right) \\
& =\omega\left(\nabla_{x} \dot{u}, J^{2}(u) \nabla_{x} \dot{u}\right) \\
& =-\omega\left(\nabla_{x} \dot{u}, \nabla_{x} \dot{u}\right)=0 .
\end{aligned}
$$

As $(N, J, h)$ has constant holomorphic sectional curvature $K$, for any tangent vectors $X, Y, Z$ and $W$, the following identity holds true (see $[\mathrm{KN}]$ ):

$$
\begin{aligned}
R(X, Y, Z, W)= & \frac{K}{4}\{h(X, Z) h(Y, W)-h(X, W) h(Y, Z)+h(X, J Z) h(Y, J W) \\
& -h(X, J W) h(Y, J Z)+2 h(X, J Y) h(Z, J W)\}
\end{aligned}
$$

Since the complex structure $J$ commutes with the Riemannian curvature operator

$$
R(X, Y) \circ J=J \circ R(X, Y)
$$


it follows from (3.2) that

$$
\begin{aligned}
\left\langle\dot{u}, J(u) R\left(u^{\prime}, \dot{u}\right) u^{\prime}\right\rangle= & \left\langle R\left(u^{\prime}, \dot{u}\right) J u^{\prime}, \dot{u}\right\rangle \\
= & R\left(u^{\prime}, \dot{u}, J u^{\prime}, \dot{u}\right) \\
= & \frac{K}{4}\left\{-\left\langle u^{\prime}, \dot{u}\right\rangle\left\langle\dot{u}, J u^{\prime}\right\rangle-\left\langle u^{\prime}, J \dot{u}\right\rangle\left\langle\dot{u}, J^{2} u^{\prime}\right\rangle\right. \\
& \left.+2\left\langle u^{\prime}, J \dot{u}\right\rangle\left\langle J u^{\prime}, J \dot{u}\right\rangle\right\} \\
= & K\left\langle u^{\prime}, \dot{u}\right\rangle\left\langle u^{\prime}, J \dot{u}\right\rangle .
\end{aligned}
$$

Thus,

$$
\frac{1}{2} \frac{d}{d t} \int_{S^{1}}|\tau(u)|^{2} d S=\int_{S^{1}} K\left\langle u^{\prime}, \dot{u}\right\rangle\left\langle u^{\prime}, J \dot{u}\right\rangle d S
$$

On the other hand, by [DW], we know that

$$
\begin{aligned}
\frac{1}{4} \frac{d}{d t} \int_{S^{1}}\left|u^{\prime}\right|^{4} d S & =\int_{S^{1}}\left|u^{\prime}\right|^{2}\left\langle u^{\prime}, \nabla_{t} u^{\prime}\right\rangle d S \\
& =\int_{S^{1}}\left|u^{\prime}\right|^{2}\left\langle u^{\prime}, \nabla_{x} \dot{u}\right\rangle d S \\
& =-\int_{S^{1}}\left\{\left|u^{\prime}\right|^{2}\left\langle\nabla_{x} u^{\prime}, \dot{u}\right\rangle+2\left\langle u^{\prime}, \dot{u}\right\rangle\left\langle u^{\prime}, \nabla_{x} u^{\prime}\right\rangle\right\} d S \\
& =-\int_{S^{1}}\left\{\left|u^{\prime}\right|^{2}\langle\tau(u), J \tau(u)\rangle+2\left\langle u^{\prime}, \dot{u}\right\rangle\left\langle u^{\prime}, \tau(u)\right\rangle\right\} d S \\
& =2 \int_{S^{1}}\left\langle u^{\prime}, \dot{u}\right\rangle\left\langle u^{\prime}, J \dot{u}\right\rangle d S
\end{aligned}
$$

Combining (3.4) and (3.5), we conclude that

$$
\frac{d}{d t}\left\{\int_{S^{1}}|\tau(u)|^{2} d S-\frac{K}{4} \int_{S^{1}}|\nabla u|^{4} d S\right\} \equiv 0 .
$$

This completes the proof of Theorem 1 .

Proof of Theorem 2. With Proposition 3.1 at hand, it remains to extend the local solution into a global one. For this, the proof proceeds similarly as in [DW] with Theorem 1 replacing Proposition 3.2 in [DW]. The issue of uniqueness has also been addressed in $[\mathrm{DW}]$.

Proof of Theorem 3. By Proposition 3.1, a maximal local smooth solution $u$ defined on $S^{1} \times\left[0, T_{\max }\right)$ exists for the Cauchy problem of the Schrödinger flow.

To prove global existence, we consider the quantity

$$
d_{\max }=\sup _{s \in S^{1}}\left\{\int_{0}^{T_{\max }}\left|\frac{d u}{d t}\right|^{2} d t\right\} .
$$

First, we note that $C H^{n}$ is a Hermitian symmetric manifold and thus its curvature tensor is parallel, i.e., $\nabla R \equiv 0$. Then, by the integrability of the complex structure 
$J$, we have

$$
\begin{aligned}
\nabla_{t} \nabla_{x} \tau(u)= & \nabla_{x} \nabla_{t} \tau(u)+R\left(u^{\prime}, \dot{u}\right) \tau(u) \\
= & \nabla_{x}\left(\nabla_{x} \nabla_{t} u^{\prime}+R\left(u^{\prime}, \dot{u}\right) u^{\prime}\right)+R\left(u^{\prime}, \dot{u}\right) \tau(u) \\
= & \nabla_{x} \nabla_{x} \nabla_{x} \dot{u}+R\left(\nabla_{x} u^{\prime}, \dot{u}\right) u^{\prime}+R\left(u^{\prime}, \nabla_{x} \dot{u}\right) u^{\prime} \\
& +R\left(u^{\prime}, \dot{u}\right) \nabla_{x} u^{\prime}+R\left(u^{\prime}, \dot{u}\right) \tau(u) \\
= & \nabla_{x} \nabla_{x} \nabla_{x} \dot{u}+R(\tau(u), J \tau(u)) u^{\prime}+R\left(u^{\prime}, J \nabla_{x} \tau(u)\right) u^{\prime} \\
& +2 R\left(u^{\prime}, \dot{u}\right) \tau(u) .
\end{aligned}
$$

Thus,

$$
\begin{aligned}
& \frac{1}{2} \frac{d}{d t} \int_{S^{1}}\left\langle\nabla_{x} \tau(u), \nabla_{x} \tau(u)\right\rangle d S \\
= & \int_{S^{1}}\left\langle\nabla_{x} \tau(u), \nabla_{t} \nabla_{x} \tau(u)\right\rangle d S \\
= & \int_{S^{1}}\left\langle\nabla_{x} \tau(u), \nabla_{x} \nabla_{x} \nabla_{x} J(u) \tau(u)\right\rangle d S \\
& +\int_{S^{1}}\left\langle R(\tau(u), J \tau(u)) u^{\prime}, \nabla_{x} \tau(u)\right\rangle d S \\
& +\int_{S^{1}}\left\langle R\left(u^{\prime}, J \nabla_{x} \tau(u)\right) u^{\prime}+2 R\left(u^{\prime}, \dot{u}\right) \tau(u), \nabla_{x} \tau(u)\right\rangle d S \\
\leq & C_{0} \int_{S^{1}}\left\{\left|u^{\prime}\right||\tau(u)|^{2}\left|\nabla_{x} \tau(u)\right|+\left|u^{\prime}\right|^{2}\left|\nabla_{x} \tau(u)\right|^{2}\right\} d S,
\end{aligned}
$$

where $C_{0}$ is a constant which is independent of $u$. By Kato inequality,

$$
\int_{S^{1}}\left|\nabla_{x}\right| u^{\prime} \|^{2} d S \leq \int_{S^{1}}\left|\nabla_{x} u^{\prime}\right|^{2} d S=\int_{S^{1}}|\tau(u)|^{2} d S
$$

and

$$
\int_{S^{1}}\left|\nabla_{x}\right| \tau(u) \|^{2} d S \leq \int_{S^{1}}\left|\nabla_{x} \tau(u)\right|^{2} d S
$$

By the Sobolev imbedding theorem,

$$
\left\|u^{\prime}\right\|_{C^{0}}^{2} \leq C\left(S^{1}\right)\left\{\int_{S^{1}}\left|\nabla_{x}\right| u^{\prime} \|^{2} d S+E(u)\right\}
$$

and

$$
\|\tau(u)\|_{C^{0}}^{2} \leq C\left(S^{1}\right)\left\{\int_{S^{1}}\left|\nabla_{x}\right| \tau(u) \|^{2} d S+\int_{S^{1}}|\tau(u)|^{2} d S\right\} .
$$

Combining the last four inequalities, we get

$$
\left\|u^{\prime}\right\|_{C^{0}}^{2} \leq C\left(S^{1}\right)\left\{\int_{S^{1}}|\tau(u)|^{2} d S+E(u)\right\}
$$

and

$$
\|\tau(u)\|_{C^{0}}^{2} \leq C\left(S^{1}\right)\left\{\int_{S^{1}}\left|\nabla_{x} \tau(u)\right|^{2} d S+\int_{S^{1}}|\tau(u)|^{2} d S\right\} .
$$


Substituting (3.10) and (3.11) into the last term on the right hand side of (3.7), in view of the fact $E(u(t)) \equiv E\left(u_{0}\right)$ and using the Hölder inequality, we have

$$
\begin{aligned}
& \int_{S^{1}}\left\{\left|u^{\prime} \| \tau(u)\right|^{2}\left|\nabla_{x} \tau(u)\right|+\left|u^{\prime}\right|^{2}\left|\nabla_{x} \tau(u)\right|^{2}\right\} d S \\
\leq & \|\tau(u)\|_{C^{0}}\left\|u^{\prime}\right\|_{C^{0}}\|\tau(u)\|_{L^{2}}\left\|\nabla_{x} \tau(u)\right\|_{L^{2}}+\left\|u^{\prime}\right\|_{C^{0}}^{2} \int_{S^{1}}\left|\nabla_{x} \tau(u)\right|^{2} d S \\
\leq & C\left(S^{1}\right)\left\{\left(\|\tau(u)\|_{L^{2}}\left\|u^{\prime}\right\|_{C^{0}}+\left\|u^{\prime}\right\|_{C^{0}}^{2}\right) \int_{S^{1}}\left|\nabla_{x} \tau(u)\right|^{2} d S+\|\tau(u)\|_{L^{2}}^{3}\left\|u^{\prime}\right\|_{C^{0}}\right\} \\
\leq & C\left(S^{1}\right)\left\{\|\tau(u)\|_{L^{2}}^{2} \int_{S^{1}}\left|\nabla_{x} \tau(u)\right|^{2} d S+\|\tau(u)\|_{L^{2}}^{3}+\sqrt{E\left(u_{0}\right)}\|\tau(u)\|_{L^{2}}^{3}\right\} \\
\leq & C\left(S^{1},\left\|\tau\left(u_{0}\right)\right\|_{L^{2}},\left\|u_{0}^{\prime}\right\|_{L^{4}}\right)\left\{\int_{S^{1}}\left|\nabla_{x} \tau(u)\right|^{2} d S+1\right\} .
\end{aligned}
$$

Here we have used the inequality

$$
\int_{S^{1}}|\tau(u)|^{2} d S \leq \int_{S^{1}}\left|\tau\left(u_{0}\right)\right|^{2} d S+\int_{S^{1}}\left|u_{0}^{\prime}\right|^{4} d S, \text { for any } t \in\left[0, T_{\max }\right),
$$

which is a consequence of the conservative law in Theorem 1, viz

$$
\int_{S^{1}}|\tau(u)|^{2} d S+\int_{S^{1}}\left|u^{\prime}(t)\right|^{4} d S \equiv \int_{S^{1}}\left|\tau\left(u_{0}\right)\right|^{2} d S+\int_{S^{1}}\left|u_{0}^{\prime}\right|^{4} d S
$$

As the holomorphic sectional curvature of $C H^{n}$ with the Bergmann metric is equal to -4 , the inequality (3.7) implies that

$$
\begin{aligned}
\frac{d}{d t} \int_{S^{1}}\left|\nabla_{x} \dot{u}\right|^{2} d S & =\frac{d}{d t} \int_{S^{1}}\left|\nabla_{x} \tau(u)\right|^{2} d S \\
& \leq C\left(S^{1},\left\|\tau\left(u_{0}\right)\right\|_{L^{2}},\left\|u_{0}^{\prime}\right\|_{L^{4}}\right)\left\{\int_{S^{1}}\left|\nabla_{x} \tau(u)\right|^{2} d S+1\right\} \\
& =C\left(S^{1},\left\|\tau\left(u_{0}\right)\right\|_{L^{2}},\left\|u_{0}^{\prime}\right\|_{L^{4}}\right)\left\{\int_{S^{1}}\left|\nabla_{x} \dot{u}\right|^{2} d S+1\right\}
\end{aligned}
$$

By the Gronwall inequality,

$$
\int_{S^{1}}\left|\nabla_{x} \dot{u}(t)\right|^{2} d S \leq\left(1+\left\|\nabla \tau\left(u_{0}\right)\right\|_{L^{2}}^{2}\right) \exp (C t)-1,
$$

where $C=C\left(S^{1},\left\|\tau\left(u_{0}\right)\right\|_{L^{2}},\left\|u_{0}^{\prime}\right\|_{L^{4}}\right)$ depends only on $u_{0}$ and $S^{1}$. Similarly, for $k \geq 1$,

$$
\int_{S^{1}}\left|\Delta^{k} \dot{u}\right|^{2} d S \leq C_{1}\left(t, k, u_{0}\right)
$$

and

$$
\int_{S^{1}}\left|\nabla_{x} \Delta^{k} \dot{u}\right|^{2} d S \leq C_{2}\left(t, k, u_{0}\right)
$$

where $C_{1}\left(t, k, u_{0}\right)$ and $C_{2}\left(t, k, u_{0}\right)$ are independent of the image set $u\left(S^{1} \times\left[0, T_{\max }\right)\right) \subset$ $N$. By the Sobolev imbedding theorem and (3.14), it follows that

$$
\|\dot{u}(t)\|_{C^{0}\left(S^{1}\right)} \leq C\left(S^{1}\right)\left\{\left(1+\left\|\nabla \tau\left(u_{0}\right)\right\|_{L^{2}}^{2}\right) \exp (C t)-1\right\}
$$


for any $t \in\left[0, T_{\max }\right)$.

Suppose $T_{\max }<\infty$, then

$$
d_{\max } \leq C\left(T_{\max }\right)<\infty .
$$

This indicates that the image set of $u$ is contained in some compact subset $\Omega \subset C H^{n}$. In this case, for a small $\sigma>0$, consider the Cauchy problem

$$
\left\{\begin{array}{l}
\frac{\partial v}{\partial t}=J(v) \tau(v) \\
v(x, 0)=u\left(x, T_{\max }-\sigma\right)
\end{array}\right.
$$

As in [DW], we can show that there exists a positive real number $T_{0}$, which depends on $\Omega$ but not on $\sigma$, such that (3.15) admits a local smooth solution $v$ on $S^{1} \times\left[0, T_{0}\right)$. Thus $u$ can be extended to $S^{1} \times\left[0, T_{\max }+T_{0}-\sigma\right)$. By the uniqeness theorem given in [DW], we know that $v(x, t)=u\left(x, T_{\max }-\sigma+t\right)$ for any $t \in\left[0, T_{0}\right)$ so the extended $u$ is still the solution for the Cauchy problem of the Schrödinger flow. Choose $\sigma$ small enough so that

$$
T_{\max }+T_{0}-\sigma>T_{\max }
$$

This contradicts the fact that $T_{\max }$ is maximal. Thus $T_{\max }$ must be $\infty$ and the proof of Theorem 3 is complete.

We end this section by recalling that the complex hyperbolic space $C H^{n}$ is a rank one symmetric space of noncompact type. Its ball model is given by $\left(B^{n}, g_{b}\right)$ where $B^{n}=\left\{z \in C^{n}:|z|<1\right\}$ and

$$
g_{b}=\frac{1}{1-|w|^{2}} \sum_{i, j=1}^{n}\left(\delta_{i j}+\frac{\bar{w}_{i} w_{j}}{1-|w|^{2}}\right) d w_{i} d \bar{w}_{j}
$$

is the Bergmann metric. It is well-known that $C H^{n}$ with the Bergmann metric is a complete Kähler manifold with holomorphic sectional curvature -4 (see e.g. [KN]). In particular, any compact Kähler manifold with constant negative holomorphic sectional curvature $-K$ must be a compact quotient of $\left(C H^{n}, d s_{K}\right)$ modulo an isometric cocompact lattice group where $d s_{K}=\frac{K}{4} g_{b}$.

4. Two-Dimensional Local Schrödinger Flow. In this section we consider Schrödinger flows defined on Riemann surfaces, and establish local existence for the Cauchy problem. As in [DW], our strategy is to consider approximate equations of Landau-Lifshitz type:

$$
\left\{\begin{array}{l}
\frac{\partial u}{\partial t}=\varepsilon \tau(u)+J(u) \tau(u) \\
u(x, 0)=u_{0}(x)
\end{array}\right.
$$

where $0<\varepsilon<1$. By the results in [Am] (see also [GW]), there exists a unique local smooth solution for the Cauchy problem (4.1) on $M \times\left[0, T^{\prime}\right)$. Also, it is well-known that the energy functional is decreasing (see e.g. [DW]), i.e., for a solution $u$ of (4.1),

$$
E(u) \leq E\left(u_{0}\right)
$$

Hereafter, we shall assume that $N$ is embedded isometrically into the Euclidean space $R^{d}$. 
The key step for proving Theorem 4 is to establish some a priori estimates for $u(x, t)$ which hold uniformly for small $\varepsilon<1$. To do this, we need the following result whose proof will be relegated to the end of the paper:

Proposition 4.1. Let $M$ be a closed Riemann surface and $N$ a compact Kähler manifold with nonpositive sectional curvature. If $u$ is a smooth solution of the Cauchy problem (4.1), then there exists a positive $T$, which does not depend on $0<\varepsilon<1$, such that the following estimates hold uniformly for $0<\varepsilon<1$ :

$$
\sup _{t \in[0, T)} \int_{M}\left|\Delta^{k} \tau(u)\right|^{2} d M \leq C\left(k, T, u_{0}\right)
$$

and

$$
\sup _{t \in[0, T)} \int_{M}\left|\nabla \Delta^{k} \tau(u)\right|^{2} d M \leq C\left(k, T, u_{0}\right)
$$

With Proposition 4.1 at hand, Theorem 4 follows immediately.

Proof of Theorem 4. By Proposition 4.1, for each $0<\varepsilon<1$, the problem (4.1) admits a unique smooth solution $u_{\varepsilon}$ on $M \times[0, T)$ where $T$ does not depend on $\varepsilon$. Note that, for any $k$,

$$
\sup _{t \in[0, T)} \int_{M}\left|\Delta^{k} \tau\left(u_{\varepsilon}\right)\right|^{2} d M \leq C\left(k, T, u_{0}\right)<\infty
$$

and

$$
\sup _{t \in[0, T)} \int_{M}\left|\nabla \Delta^{k} \tau\left(u_{\varepsilon}\right)\right|^{2} d M \leq C\left(k, T, u_{0}\right)<\infty .
$$

Now letting $\varepsilon \rightarrow 0$, it follows from the Sobolev imbedding theorem that there exists a function $u(x, t)$ defined on $M \times[0, T)$ such that, for any $T^{\prime \prime}<T$ and any $k \geq 1$,

$$
\left\|u_{\varepsilon}-u\right\|_{C^{2 k, k}\left(M \times\left[0, T^{\prime \prime}\right], R^{d}\right)} \longrightarrow 0
$$

where $\|\cdot\|_{C^{2 k, k}\left(M \times\left[0, T^{\prime \prime}\right], R^{d}\right)}$ denotes the parabolic Hölder norm. Furthermore, $u(x, t)$ satisfies (1.1). This completes the proof of Theorem 4.

In order to prove Proposition 4.1, the following two lemmas are needed:

Lemma 4.2. Let $M$ be a closed Riemann surface and $N$ a compact Kähler manifold with nonpositive sectional curvature. If $u$ is a smooth solution of the Cauchy problem (4.1), then there exists a positive $T$, which is independent of $0<\varepsilon<1$, such that the following inequality holds uniformly for $0<\varepsilon<1$ :

$$
\sup _{t \in[0, T)} \int_{M}|\nabla \tau(u)|^{2} d M \leq C\left(T, u_{0}\right)
$$

Proof. For ease of notation, denote $\frac{\partial u}{\partial t}$ by $\dot{u}$, and $u_{*} e_{i}$ by $u_{i}$. As $\nabla J \equiv 0$, by a 
direct calculation,

$$
\begin{aligned}
\frac{1}{2} \frac{d}{d t} \int_{M}|\nabla \dot{u}|^{2} d M & =\int_{M}\left\langle\nabla_{i} \dot{u}, \nabla_{t} \nabla_{i} \dot{u}\right\rangle d M \\
& =\int_{M}\left\langle\nabla_{i} \dot{u}, \nabla_{t} \nabla_{i}(\varepsilon \tau(u)+J(u) \tau(u))\right\rangle d M \\
& =\int_{M}\left\{\varepsilon\left\langle\nabla_{i} \dot{u}, \nabla_{t} \nabla_{i} \nabla_{j} u_{j}\right\rangle+\left\langle\nabla_{i} \dot{u}, J(u) \nabla_{t} \nabla_{i} \nabla_{j} u_{j}\right\rangle\right\} d M .
\end{aligned}
$$

By the commutation relation of covariant derivatives, we have the following formula:

$$
\begin{aligned}
\nabla_{t} \nabla_{i} \nabla_{j} u_{j}= & \nabla_{i} \nabla_{t} \nabla_{j} u_{j}+R\left(u_{i}, \dot{u}\right) \nabla_{j} u_{j} \\
= & \nabla_{i} \nabla_{j} \nabla_{t} u_{j}+\nabla_{i}\left(R\left(u_{j}, \dot{u}\right) u_{j}\right)+R\left(u_{i}, \dot{u}\right) \nabla_{j} u_{j} \\
= & \nabla_{i} \nabla_{j} \nabla_{j} \dot{u}+R\left(u_{i}, \dot{u}\right) \nabla_{j} u_{j}+R\left(\nabla_{i} u_{j}, \dot{u}\right) u_{j} \\
& +R\left(u_{j}, \nabla_{i} \dot{u}\right) u_{j}+R\left(u_{j}, \dot{u}\right) \nabla_{i} u_{j}+\left(\nabla_{i} R\right)\left(u_{j}, \dot{u}\right) u_{j} .
\end{aligned}
$$

Combining (4.2) and (4.3), we obtain that, for small $\varepsilon$,

$$
\begin{aligned}
\frac{1}{2} \frac{d}{d t} \int_{M}|\nabla \dot{u}|^{2} d M \leq & -\varepsilon \int_{M}\langle\Delta \dot{u}, \Delta \dot{u}\rangle d M+C(M, N)\left\{\int _ { M } \left(|\nabla \dot{u} \| \tau(u)|^{2}|d u|\right.\right. \\
& \left.\left.+\left.|\nabla \dot{u}\|\tau(u)\| d u \|| \nabla d u|+| d u\right|^{2}|\nabla \dot{u}|^{2}+|\nabla \dot{u}||d u|^{3}|\dot{u}|\right) d M\right\} \\
\leq & -\varepsilon \int_{M}\langle\Delta \dot{u}, \Delta \dot{u}\rangle d M+C(M, N)\left\{\int _ { M } \left(|d u|^{3}|\dot{u}||\nabla \dot{u}|\right.\right. \\
& \left.\left.+|\nabla \dot{u}|\left\{|\nabla d u|^{2}|d u|+|\nabla \dot{u} \| d u|^{2}\right\}\right) d M\right\} .
\end{aligned}
$$

Here we have used $|\tau(u)|^{2} \leq C(M, N)|\nabla d u|^{2}$ and

$$
\int_{M}\left\langle\nabla_{i} \dot{u}, J(u)\left(\nabla_{i} \nabla_{j} \nabla_{j} \dot{u}\right)\right\rangle d M=-\int_{M}\langle\Delta \dot{u}, J(u) \Delta \dot{u}\rangle d M=0 .
$$

From (2.10), we have the estimate

$$
\|d u\|_{C^{0}} \leq C\left(M, N, E\left(u_{0}\right)\right)\left\{\left(\int_{M}|\nabla \tau(u)|^{2} d M\right)^{\frac{1}{2}}+\sqrt{E(u)}\right\} .
$$

Upon differentiating the two sides of (4.1),

$$
\nabla \dot{u}=\varepsilon \nabla \tau(u)+J(u) \nabla \tau(u),
$$

and as $\langle\nabla \tau(u), J(u) \nabla \tau(u)\rangle \equiv 0$, we have

$$
|\nabla \dot{u}|^{2}=\left(1+\varepsilon^{2}\right)|\nabla \tau(u)|^{2} .
$$

Thus, it follows that

$$
\|d u\|_{C^{0}} \leq \frac{C(M, N)}{1+\varepsilon^{2}}\left\{\left(\int_{M}|\nabla \dot{u}|^{2} d M\right)^{\frac{1}{2}}+\sqrt{E(u)}\right\} .
$$

By Propositions 2.3 and 2.5 and using (2.4),

$$
\begin{aligned}
\|\nabla d u\|_{L^{4}}^{2} & \leq C(M, N)\left\{\|d u\|_{W^{1,4}}^{2}+\|d u\|_{L^{8}}^{4}\right\} \\
& \leq C(M, N)\left\{\|d u\|_{W^{1,4}}^{2}+\|d u\|_{L^{2}}^{3}\left(\|\nabla d u\|_{L^{2}}+\sqrt{E\left(u_{0}\right)}\right)\right\} \\
& \leq C(M, N)\left\{\|d u\|_{W^{1,4}}^{2}+\|d u\|_{L^{2}}^{3}\left(\|\tau(u)\|_{L^{2}}+C \sqrt{E\left(u_{0}\right)}\right)\right\} \\
& \leq C(M, N)\left\{\|d u\|_{W^{2,2}}^{\frac{3}{2}}\|d u\|_{L^{2}}^{\frac{1}{2}}+\|d u\|_{L^{2}}^{3}\left(\|\tau(u)\|_{L^{2}}+C \sqrt{E\left(u_{0}\right)}\right)\right\} \\
& \leq C(M, N, E(u))\left\{\|\nabla \tau(u)\|_{L^{2}}+\|\nabla \tau(u)\|_{L^{2}}^{\frac{3}{2}}+1\right\}^{\frac{3}{2}}
\end{aligned}
$$


Next, we use (2.4) and (4.5)-(4.6) (see also (2.6)) to estimate the last term on the right hand side of (4.4). By the Hölder inequality, we obtain that

$$
\begin{aligned}
& \int_{M}|\nabla \dot{u}|\left\{|d u|^{3}|\dot{u}|+|\nabla d u|^{2}|d u|+|\nabla \dot{u} \| d u|^{2}\right\} d M \\
\leq & \|d u\|_{C^{0}}^{2}\left(\|d u\|_{C^{0}}\|\dot{u}\|_{L^{2}}\|\nabla \dot{u}\|_{L^{2}}+\|\nabla \dot{u}\|_{L^{2}}^{2}+\|d u\|_{C^{0}}\|\nabla d u\|_{L^{4}}^{2}\|\nabla \dot{u}\|_{L^{2}}\right. \\
\leq & C\left(M, N, E\left(u_{0}\right)\right)\left\{\|\nabla \dot{u}\|_{L^{2}}^{4}+\|\nabla \dot{u}\|_{L^{2}}^{\frac{9}{2}}+\left(\|\nabla \dot{u}\|_{L^{2}}+\|\nabla \dot{u}\|_{L^{2}}^{\frac{3}{2}}+1\right)^{\frac{7}{2}}\right\} \\
\equiv & \tilde{F}\left(\|\nabla \dot{u}\|_{L^{2}}^{2}\right) .
\end{aligned}
$$

Thus, by (4.4) and (4.7),

$$
\frac{d}{d t}\|\nabla \dot{u}\|_{L^{2}}^{2} \leq \tilde{F}\left(\|\nabla \dot{u}\|_{L^{2}}^{2}\right)
$$

It follows from the last inequality that, for $0<\varepsilon<1$,

$$
\begin{aligned}
\frac{d}{d t}\|\nabla \tau(u)\|_{L^{2}}^{2} & \leq \frac{1}{1+\varepsilon^{2}} \tilde{F}\left(\left(1+\varepsilon^{2}\right)\|\nabla \tau(u)\|_{L^{2}}^{2}\right) \\
& \leq \tilde{F}\left(2\|\nabla \tau(u)\|_{L^{2}}^{2}\right) \equiv F\left(\|\nabla \tau(u)\|_{L^{2}}^{2}\right)
\end{aligned}
$$

since

$$
|\nabla \dot{u}|^{2}=\left(1+\varepsilon^{2}\right)|\nabla \tau(u)|^{2} .
$$

We note that $F(\cdot)$ depends on $M, N$ and $E\left(u_{0}\right)$, and not on $\varepsilon$.

Consider now the initial value problem for the ordinary differential equation

$$
\left\{\begin{array}{l}
\frac{d q(t)}{d t}=F(q(t)), \\
q(0)=\int_{M}\left|\nabla \tau\left(u_{0}\right)\right|^{2} d M .
\end{array}\right.
$$

It is easy to see that this initial value problem has a local smooth solution. Hence, by the comparison principle of ordinary differential equations, there exists a positive real number $T$, which does not depend on $\varepsilon$, such that on $[0, T)$

$$
\sup _{t \in[0, T)} \int_{M}|\nabla \tau(u)|^{2} d M \leq C\left(T, u_{0}\right),
$$

where $C\left(T, u_{0}\right)$ is independent of $\varepsilon$. This finishes the proof of Lemma 4.2.

LEMMA 4.3. Under the same assumptions as in Lemma 4.2, let $u$ be a smooth solution of the Cauchy problem (4.1). Then the following estimate holds for $t \in[0, T)$ uniformly for $0<\varepsilon<1$ and $k \geq 1$ :

$$
\left\|\nabla^{k+1} d u\right\|_{L^{2}} \leq\|d u\|_{W^{k+1,2}}+C\left(\|d u\|_{W^{k, 2}},\|\nabla \tau(u)\|_{L^{2}}, E\left(u_{0}\right)\right) .
$$

Proof. Using the estimate

$$
\|d u\|_{C^{0}\left(M, R^{d}\right)} \leq C\left(\|\nabla \tau(u)\|_{L^{2}}, E\left(u_{0}\right)\right)
$$

and the expression of $\nabla^{k+1} d u$ in a local coordinate chart, the proof proceeds as that of Proposition 2.4. We will omit the details. 
We complete this paper with the proof of Proposition 4.1.

Proof of Proposition 4.1. The proof proceeds by induction. First, we consider the case of $k=1$. We compute

$$
\begin{aligned}
& \frac{1}{2} \frac{d}{d t} \int_{M}|\Delta \dot{u}|^{2} d M \\
= & \int_{M}\left\langle\nabla_{j} \nabla_{j} \dot{u}, \nabla_{t} \nabla_{i} \nabla_{i} \dot{u}\right\rangle d M \\
= & \int_{M}\left\{\varepsilon\left\langle\Delta \dot{u}, \nabla_{t} \Delta \tau(u)\right\rangle+\left\langle\nabla_{j} \nabla_{j} \dot{u}, J(u) \nabla_{t} \nabla_{i} \nabla_{i} \tau(u)\right\rangle\right\} d M .
\end{aligned}
$$

Now, we note that

$$
\begin{aligned}
& \nabla_{t} \nabla_{i} \nabla_{i} \tau(u) \\
= & \nabla_{i} \nabla_{t} \nabla_{i} \tau(u)+R\left(u_{i}, \dot{u}\right) \nabla_{i} \tau(u) \\
= & \nabla_{i} \nabla_{i} \nabla_{t} \tau(u)+\nabla_{i}\left(R\left(u_{i}, \dot{u}\right) \tau(u)\right)+R\left(u_{i}, \dot{u}\right) \nabla_{i} \tau(u) \\
= & \nabla_{i} \nabla_{i} \nabla_{j} \nabla_{j} \dot{u}+\nabla_{i} \nabla_{i}\left(R\left(u_{j}, \dot{u}\right) u_{j}\right)+\nabla_{i}\left(R\left(u_{i}, \dot{u}\right) \tau(u)\right) \\
& +R\left(u_{i}, \dot{u}\right) \nabla_{i} \tau(u) .
\end{aligned}
$$

Also, as in (4.3), we have

$$
\begin{aligned}
\nabla_{i}\left(R\left(u_{i}, \dot{u}\right) \tau(u)\right)= & R(\tau(u), \dot{u}) \tau(u)+R\left(u_{i}, \nabla_{i} \dot{u}\right) \tau(u) \\
& +R\left(u_{i}, \dot{u}\right) \nabla_{i} \tau(u)+\left(\nabla_{i} R\right)\left(u_{i}, \dot{u}\right) \tau(u)
\end{aligned}
$$

and

$$
\begin{aligned}
& \nabla_{i} \nabla_{i}\left(R\left(u_{j}, \dot{u}\right) u_{j}\right) \\
= & \nabla_{i}\left\{\left(\nabla_{i} R\right)\left(u_{j}, \dot{u}\right) u_{j}+R\left(\nabla_{i} u_{j}, \dot{u}\right) u_{j}+R\left(u_{j}, \nabla_{i} \dot{u}\right) u_{j}+R\left(u_{j}, \dot{u}\right) \nabla_{i} u_{j}\right\} \\
= & R\left(\Delta u_{j}, \dot{u}\right) u_{j}+R\left(\nabla_{i} u_{j}, \nabla_{i} \dot{u}\right) u_{j}+R\left(\nabla_{i} u_{j}, \dot{u}\right) \nabla_{i} u_{j} \\
& +R\left(\nabla_{i} u_{j}, \nabla_{i} \dot{u}\right) u_{j}+R\left(u_{j}, \Delta \dot{u}\right) u_{j}+R\left(u_{j}, \nabla_{i} \dot{u}\right) \nabla_{i} u_{j} \\
& +R\left(\nabla_{i} u_{j}, \dot{u}\right) \nabla_{i} u_{j}+R\left(u_{j}, \nabla_{i} \dot{u}\right) \nabla_{i} u_{j}+R\left(u_{j}, \dot{u}\right) \Delta u_{j}+\left(\nabla_{i} \nabla_{i} R\right)\left(u_{j}, \dot{u}\right) u_{j} \\
& +2\left(\nabla_{i} R\right)\left(\nabla_{i} u_{j}, \dot{u}\right) u_{j}+2\left(\nabla_{i} R\right)\left(u_{j}, \nabla_{i} \dot{u}\right) u_{j}+2\left(\nabla_{i} R\right)\left(u_{j}, \dot{u}\right) \nabla_{i} u_{j} .
\end{aligned}
$$

Substituting (4.10)-(4.12) into (4.9) and using the estimate

$$
\|d u\|_{C^{0}} \leq C(M, N)\left\{\|\nabla \tau(u)\|_{L^{2}}+\sqrt{E\left(u_{0}\right)}\right\} \leq C\left(T, u_{0}\right)
$$

we obtain that

$$
\begin{aligned}
& \frac{d}{d t} \int_{M}|\Delta \dot{u}|^{2} d M+2 \varepsilon \int_{M}|\nabla \Delta \dot{u}|^{2} d M \\
(4.13)= & C\left(M, N, T, u_{0}\right)\left\{\int_{M}|\tau(u)||\Delta \dot{u}|\left\{|\tau(u)|^{2}+|\nabla \tau(u)|+\left|\nabla^{2} d u\right|\right\} d M\right. \\
& \left.+\int_{M}|\Delta \dot{u}|\left\{|\Delta \dot{u}|+|\nabla \tau(u)||\nabla d u|+|\nabla d u|^{2}|\tau(u)|+|\nabla d u|^{2}+|\nabla \dot{u}|\right\} d M\right\} .
\end{aligned}
$$

Thus, by using the Hölder inequality on the integral terms on the right hand side of 
(4.13), it follows that

$$
\begin{aligned}
& C\left(M, N, T, u_{0}\right)\left\{\int_{M}|\tau(u) \| \Delta \dot{u}|\left\{|\tau(u)|^{2}+|\nabla \tau(u)|+\left|\nabla^{2} d u\right|\right\} d M\right. \\
& \left.+\int_{M}|\Delta \dot{u}|\left\{|\Delta \dot{u}|+|\nabla \tau(u) \| \nabla d u|+|\nabla d u|^{2}|\tau(u)|+|\nabla d u|^{2}+\left|\nabla_{i} \dot{u}\right|\right\} d M\right\} \\
\leq & C\left(M, N, T, u_{0}\right)\left\{\|\Delta \dot{u}\|_{L^{2}}^{2}\|\tau(u)\|_{L^{6}}^{3}+\|\tau(u)\|_{C^{0}}\|\nabla \tau(u)\|_{L^{2}}\|\Delta \dot{u}\|_{L^{2}}\right. \\
& +\|\tau(u)\|_{C^{0}}\left\|\nabla^{2} d u\right\|_{L^{2}}\|\Delta \dot{u}\|_{L^{2}}+\|\Delta \dot{u}\|_{L^{2}}^{2}+\|\Delta \dot{u}\|_{L^{2}}\|\nabla d u\|_{C^{0}}\|\nabla \tau(u)\|_{L^{2}} \\
& \left.+\|\Delta \dot{u}\|_{L^{2}}\left(\|\nabla d u\|_{L^{6}}^{3}+\|\nabla d u\|_{C^{0}}\|\nabla d u\|_{L^{2}}+\|\nabla \dot{u}\|_{L^{2}}\right)\right\} .
\end{aligned}
$$

By the interpolation inequality, we have

$$
\|\tau(u)\|_{L^{6}} \leq C(M, N)\|\tau(u)\|_{H^{1,2}}^{\frac{2}{3}}\|\tau(u)\|_{L^{2}}^{\frac{1}{3}} .
$$

The Sobolev imbedding theorem and Proposition 2.4 imply that

$$
\|\tau(u)\|_{C^{0}} \leq C\left\{\|\Delta \tau(u)\|_{L^{2}}+C(M, N)\left(\|\nabla \tau(u)\|_{L^{2}}, E\left(u_{0}\right)\right)\right\},
$$

and

$$
\|\nabla d u\|_{C^{0}} \leq C(M, N)\left\{\|\Delta \tau(u)\|_{L^{2}}+C\left(\|\nabla \tau(u)\|_{L^{2}}, E\left(u_{0}\right)\right)\right\} .
$$

Further, by Lemma 4.3 and Proposition 2.3,

$$
\begin{aligned}
\|d u\|_{H^{2,2}} & \leq\|d u\|_{W^{2,2}}+C^{\prime}\left(\|\nabla \tau(u)\|_{L^{2}}, E\left(u_{0}\right)\right) \\
& \leq C\left(\|\nabla \tau(u)\|_{L^{2}}, E\left(u_{0}\right)\right)=C\left(T, u_{0}\right) .
\end{aligned}
$$

Using this esimate, the Kato inequality and Propsition 2.5, we obtain that

$$
\begin{aligned}
\|\nabla d u\|_{L^{6}} & \leq C(M, N)\left\{\|\nabla d u\|_{L^{2}}+\|\nabla \mid \nabla d u\|_{L^{2}}\right\}^{\frac{2}{3}}\|\nabla d u\|_{L^{2}}^{\frac{1}{3}} \\
& \leq C(M, N)\left\{\|\nabla d u\|_{L^{2}}+\left\|\nabla^{2} d u\right\|_{L^{2}}\right\}^{\frac{2}{3}}\|\nabla d u\|_{L^{2}}^{\frac{1}{3}} \\
& \leq C(M, N)\|d u\|_{H^{2,2}}^{\frac{2}{3}}\|\nabla d u\|_{L^{2}}^{\frac{1}{3}} \\
& \leq C\left(M, N,\|\nabla \tau(u)\|_{L^{2}}, E\left(u_{0}\right)\right) \\
& \leq C\left(T, u_{0}\right)
\end{aligned}
$$

Finally, substituting (2.4), (4.15)-(4.18) into (4.14), we get

$$
\frac{d}{d t} \int_{M}|\Delta \dot{u}|^{2} d M=-2 \varepsilon \int_{M}|\nabla \Delta \dot{u}|^{2} d M+C\left(T, u_{0}\right)\left\{\int_{M}|\Delta \dot{u}|^{2} d M+1\right\} .
$$

It follows that

$$
\int_{M}|\Delta \dot{u}|^{2} d M \leq C\left(T, u_{0}\right) \leq \infty, \quad t \in[0, T),
$$

and hence

$$
\int_{M}|\Delta \tau(u)|^{2} d M \leq C\left(T, u_{0}\right) \leq \infty, \quad t \in[0, T) .
$$

For the induction step, assuming

$$
\int_{M}\left|\Delta^{k} \tau(u)\right|^{2} d M \leq C\left(k, T, u_{0}\right), \quad t \in[0, T)
$$


for $k \geq 1$, we would like to prove

$$
\sup _{t \in[0, T)} \int_{M}\left|\nabla \Delta^{k} \tau(u)\right|^{2} d M \leq C\left(k, T, u_{0}\right)
$$

As before, we consider

$$
\begin{aligned}
& \frac{1}{2} \frac{d}{d t} \int_{M}\left|\nabla \Delta^{k} \dot{u}\right|^{2} d M \\
= & \int_{M}\left\langle\nabla_{i} \Delta^{k} \dot{u}, \nabla_{t} \nabla_{i} \Delta^{k} \dot{u}\right\rangle d M \\
= & \int_{M}\left\{\varepsilon\left\langle\nabla_{i} \Delta^{k} \dot{u}, \nabla_{t} \nabla_{i} \Delta^{k} \tau(u)\right\rangle+\left\langle\nabla_{i} \Delta^{k} \dot{u}, J(u) \nabla_{t} \nabla_{i} \Delta^{k} \tau(u)\right\rangle\right\} d M .
\end{aligned}
$$

It is not difficult to see from the commutation relation of covariant derivatives as in the previous argument that

$$
\begin{aligned}
\nabla_{t} \nabla_{i} \Delta^{k} \tau(u)= & \sum \nabla_{p_{1}} \cdots \nabla_{p_{s_{0}}} R\left(\nabla_{i_{1}} \cdots \nabla_{i_{s_{1}}} u, \nabla_{j_{1}} \cdots \nabla_{j_{s_{2}}} \dot{u}\right) \nabla_{k_{1}} \cdots \nabla_{k_{s_{3}}} u \\
& +\nabla_{i} \Delta^{k+1} \dot{u}
\end{aligned}
$$

where $s_{0}+s_{1}+s_{2}+s_{3}=2 k+3$, and up to a permutation

$$
\left(p_{1}, \cdots, p_{s_{0}}, i_{1}, \cdots, i_{s_{1}}, j_{1}, \cdots, j_{s_{2}}, k_{1}, \cdots, k_{s_{3}}\right)=\left(i, l_{1}, l_{1}, \cdots, l_{k+1}, l_{k+1}\right), \quad l_{i}=1 \text { or } 2 .
$$

We can rewrite $(4.20)$ as

$$
\begin{aligned}
\nabla_{t} \nabla_{i} \Delta^{k} \tau(u)= & \nabla_{i} \Delta^{k+1} \dot{u}+R\left(u_{j}, \nabla_{i} \Delta^{k} \dot{u}\right) u_{j}+\mathcal{Q}_{1}\left(d u, \nabla d u, \nabla^{2 k+1} d u\right) \\
& +\mathcal{Q}_{2}\left(d u, \nabla d u, \cdots, \nabla^{2 k} d u\right),
\end{aligned}
$$

where $\mathcal{Q}_{1}, \mathcal{Q}_{2}$ are multilinear vector-valued functions and

$$
\begin{aligned}
& \left|\mathcal{Q}_{2}\left(d u, \nabla d u, \cdots, \nabla^{2 k+1} d u\right)\right| \\
\leq & \begin{cases}C(N)\left|\nabla^{2} d u\right|^{2}|d u|+\mathcal{P}\left(|d u|,|\nabla d u|,\left|\nabla^{2} d u\right|\right), & k=1, \\
C(N)\left|\nabla^{2 k} d u\right| \mathcal{P}^{\prime}\left(|d u|,|\nabla d u|,\left|\nabla^{2} d u\right|\right)+\mathcal{P}\left(|d u|, \cdots,\left|\nabla^{2 k-1} d u\right|\right), & k>1,\end{cases}
\end{aligned}
$$

for some positive multilinear functions $\mathcal{P}^{\prime}$ and $\mathcal{P}$. Thus, from (4.21) and (4.22) we have

$$
\begin{aligned}
& \left|\left\langle\nabla_{i} \Delta^{k} \dot{u}, J(u) \nabla_{t} \nabla_{i} \Delta^{k} \tau(u)\right\rangle\right| \\
= & \left|\left\langle\nabla_{i} \Delta^{k} \dot{u}, J(u) \sum R\left(\nabla_{i_{1}} \cdots \nabla_{i_{s_{1}}} u, \nabla_{j_{1}} \cdots \nabla_{j_{s_{2}}} \dot{u}\right) \nabla_{k_{1}} \cdots \nabla_{k_{s_{3}}} u\right\rangle\right| \\
\leq \quad & \left|\left\langle\nabla_{i} \Delta^{k} \dot{u}, J(u)\left\{R\left(u_{j}, \nabla_{i} \Delta^{k} \dot{u}\right) u_{j}+\mathcal{Q}_{1}\left(d u, \nabla d u, \nabla^{2 k+1} d u\right)\right\}\right\rangle\right| \\
& +\left|\left\langle\nabla_{i} \Delta^{k} \dot{u}, J(u) \mathcal{Q}_{2}\left(d u, \nabla d u, \cdots, \nabla^{2 k} d u\right)\right\rangle\right| .
\end{aligned}
$$

When $k=1$, applying Lemma 4.3, Proposition 2.4 and the Sobolev imbedding theorem, we obtain from (4.19) and (4.23) that

$$
\begin{aligned}
& \frac{1}{2} \frac{d}{d t} \int_{M}|\nabla \Delta \dot{u}|^{2} d M \\
\leq & -\varepsilon \int_{M}\left|\Delta^{2} \dot{u}\right|^{2} d M+C\left(N, u_{0}\right) \int_{M}\left\{|\nabla \Delta \dot{u}|^{2}+|\nabla \Delta \dot{u}|\left|\nabla^{3} d u\right|\right. \\
& \left.+|\nabla \Delta \dot{u}|\left|\nabla^{2} d u\right|^{2}+\left|\nabla \Delta^{k} \dot{u}\right| \mathcal{P}\left(|d u|,|\nabla d u|,\left|\nabla^{2} d u\right|\right)\right\} d M \\
\leq & -\varepsilon \int_{M}\left|\Delta^{2} \dot{u}\right|^{2} d M+C\left(N, u_{0}\right) \int_{M}|\nabla \Delta \dot{u}|^{2} d M+C\left(M, N, u_{0}\right) .
\end{aligned}
$$


When $k>1$, by using Proposition 2.4, Lemma 4.3 and the Sobolev imbedding theorem, the following two inequalities follow:

$$
\begin{aligned}
\int_{M}\left\{\mathcal{P}\left(|d u|, \cdots,\left|\nabla^{2 k-1} d u\right|\right)\right\}^{2} d M & \leq C\left(N^{\prime},\left\|u_{0}\right\|_{W^{2 k+2,2}}\right) \\
& \leq C\left(\Lambda^{\prime},\left\|\Delta^{k} \tau\left(u_{0}\right)\right\|_{L^{2}}, \cdots,\left\|\nabla \tau\left(u_{0}\right)\right\|_{L^{2}}, E\left(u_{0}\right)\right)
\end{aligned}
$$

and

$$
\begin{aligned}
\left\|\mathcal{P}^{\prime}\left(|d u|,|\nabla d u|,\left|\nabla^{2} d u\right|\right)\right\|_{C^{0}} & \leq C\left(N,\left\|u_{0}\right\|_{W^{-5.2}}\right) \\
& \leq C\left(N,\left\|\Delta^{k} \tau\left(u_{0}\right)\right\|_{L^{2}}, \cdots,\left\|\nabla \tau\left(u_{0}\right)\right\|_{L^{2}}, E\left(u_{0}\right)\right) .
\end{aligned}
$$

Therefore, by a direct calculation, we deduce from (4.19) and (4.23) that

$$
\begin{aligned}
& \frac{1}{2} \frac{d}{d t} \int_{M}\left|\nabla \Delta^{k} \dot{u}\right|^{2} d M \\
\leq & -\varepsilon \int_{M}\left|\Delta^{k+1} \dot{u}\right|^{2} d M+C\left(N, u_{0}\right) \int_{M}\left\{\left|\nabla \Delta^{k} \dot{u}\right|^{2}+\left|\nabla \Delta^{k} \dot{u} \| \nabla^{2 k+2} u\right|\right. \\
& \left.+\left|\nabla \Delta^{k} \dot{u}\right|\left|\nabla^{2 k+1} u\right|+\left|\nabla \Delta^{k} \dot{u}\right| \mathcal{P}\left(|d u|, \cdots,\left|\nabla^{2 k-1} d u\right|\right)\right\} d M \\
\leq & -\varepsilon \int_{M}\left|\Delta^{k+1} \dot{u}\right|^{2} d M+C\left(N, u_{0}\right) \int_{M}\left|\nabla \Delta^{k} \dot{u}\right|^{2} d M+C\left(M, N, u_{0}\right),
\end{aligned}
$$

where $C\left(N, u_{0}\right)$ and $C\left(M, N, u_{0}\right)$ depend on $E\left(u_{0}\right),\left\|\tau\left(u_{0}\right)\right\|_{L^{2}}, \cdots,\left\|\Delta^{k} \tau\left(u_{0}\right)\right\|_{L^{2}}$. By the Gronwall inequality the required estimate follows from (4.24) or (4.25). Similarly, we can also deduce

$$
\sup _{t \in[0, T)} \int_{M}\left|\Delta^{k+1} \tau(u)\right|^{2} d M \leq C\left(k+1, T, u_{0}\right),
$$

where $C\left(k+1, T, u_{0}\right)$ depends on $E\left(u_{0}\right),\left\|\tau\left(u_{0}\right)\right\|_{L^{2}}, \cdots,\left\|\Delta^{k+1} \tau\left(u_{0}\right)\right\|_{L^{2}}$. This completes the proof of Proposition 4.1.

\section{REFERENCES}

[Am] H. AmANn, Quasilinear parabolic systems under nonlinear boundary conditions, Arch. Rat. Mech. Anal., 92 (1986), pp. 153-192.

[Au] T. Aubin, Some Nonlinear Problems in Riemannian Geometry, Springer-Verlag, BerlinHeidelberg-New York, 1998.

[B] J. BouRgaIn, Fourier transform restriction phenomena for certain lattice subsets and applications to nonlinear evolution equations, Geom. Funct. Anal., 3(1993), pp. 107156.

[B1] J. BourgaIN, Exponential sums and nonlinear Schrödinger equations, Geom. Funct. Anal. 3 (1993), pp. 157-178.

[CSU] N. Chang, J. Shatah, And K. Uhlenbeck, Schrödinger maps, Comm. Pure Appl. Math., $53(2000)$, pp. 590-602.

[Ch] H. ChIHARA, Local existence for semilinear Schrödinger equation, Math. Japonica, 42 (1995), pp. 35-52.

[Cz] T. CAzenave, An Introduction to Nonlinear Schrödinger Equations, Textos de Métodos Matemáticos 22, Universidade Federal do Rio de Janeiro, 1989.

[D] Q. DiNG, A note on NLS and the Schrödinger flow of maps, Phys. Lett. A, 248 (1998), pp. $49-54$.

[DK] S. K. Donaldson And P. B. Kronhemier, The Geometry of Four-Manifolds, Oxford University Press, Oxford, 1990.

[DPL] . M. DANiel, K. Porsezian, AND M. LAKshmanan, On the integrability of the inhomogeneous spherically symmetric Heisenberg ferromagnet in arbitrary dimension, J. Math. Phys., 35 (1994), pp. 6498-6510. 
[DW] W. Y. DING AND Y. D. WANG, Schrödinger flows of maps into symplectic manifolds, Science in China A, 41:7 (1998), pp. 746-755.

[EL] J. Eells ANd L. Lemaire, Another report on harmonic maps, Bull. London Math. Soc., 20 (1988), pp. 385-524.

[F] A. FreIRE, Global weak solutions of the wave map system to compact homogeneous spaces, Manuscripta Math., 91 (1996), pp. 525-533.

[FT] L. Faddeev and L. A. Takhtajan, Hamiltonian Methods in the Theory of Solitons, Springer-Verlag, Berlin-Heidelberg-New York, 1987.

[G] M. GRILLAKIS, Regularity and asymptotic behavior of the wave equation with critical nonlinearity, Ann. Math., 132 (1990), pp. 485-509.

[GH] B. GoU AND M. HoNG, The Landau-Lifshitz equation of the ferromagnetic spin chain and harmonic maps, Calc. Var. Partial Differential Equations, 1 (1993), pp. 311-334.

[GV] J. Ginibre AND G. Velo, Scattering theory in the energy space for a class of nonlinear Schrödinger equations, J. Math. Pure Appl., 64 (1985), pp. 363-401.

[GW] B. GUo AND Y. D. WANG, Generalized Landau-Lifshitz systems of ferromagnetic spin chain type and harmonic maps, Science in China A, 39:12 (1996), pp. 1242-1257.

[H] R. S. Hamilton, Harmonic Maps of Manifolds with Boundary, LNM 471, Springer-Verlag, Berlin-Heidelberg-New York, 1975.

[HH] N. HAYASHI AND H. HiRATA, Global existence of small solutions to nonlinear Schrödinger equations, Nonlinear Anal., 31 (1998), pp. 671-685.

[HO] N. HAYASH AND T. OzAWA, Remarks on nonlinear Schrödinger equations in one space dimension, Differential Integral Equations, 7 (1994), pp. 453-461.

[KPV] C. E. Kenig, G. Ponce, ANd L. VeGA, Small solutions to nonlinear Schrödinger equations, Anal. Nonlinéare, 10 (1993), pp. 255-288.

[KPV1] C. E. Kenig, G. Ponce, AND L. VeGA, Smoothing effects and local existence theory for the generalized nonlinear Schrödinger equations, Invent. Math., 134 (1998), pp. 44894545 .

[KN] S. Kobayashi and K. Nomizu, Foundations of Differential Geometry, vol. 1, Interscience Publishers, New York, 1963.

[L] M. LAKSHMANAN, Continuum spin system as an exactly solvable dynamical system, Phys. Lett. A, 61 (1977), pp. 53-54.

[LL] L. D. LANDAU AND E. M. Lifshitz, On the theory of the dispersion of magnetic permeability in ferromagnetic bodies, Phys. Z. Sowj., 8 (1935), pp. 153-167; reproduced in Collected Papers of L. D. Landau, Pergaman Press, New York, 1965, pp. 101-114.

[LN] M. LAKSHMANAN AND K. NAKAMURA, Landau-Lifshitz equation of ferromagnetism, exact treatment of the Gilbert damping, Phys. Rev. Lett., 53:26 (1984), pp. 2497-2499.

[LP] M. Lakshamanan and K. PorsezIAN, Planar radially symmetric Heisenberg spin system and generalized nonlinear Schrödinger equations: Gauge equivalence, Bäckland transformations and explicit solutions, Phys. Lett. A, 146 (1990), pp. 329-334.

[MS] S. MÜllER AND M. STRUWE, Global existence of wave maps in $1+2$ dimensions with finite energy data, Topol. Methods Nonlinear Anal., 7 (1996), pp. 245-259.

[NS] K. NAKAmURA AND T. SASADA, Soliton and wave trains in ferromagnets, Phys. Lett. A, 48 (1974), pp. 321-322.

[PWW] P. Y. H. PANG, H. Y. WANG, AND Y. D. WANG, Local existence for inhomogeneous Schrödinger flow into Kähler manifolds, Acta Math. Sinica, Eng. Ser., 16 (2000), pp. 487-504.

[PT] N. Papanicolaou and T. Thomaras, Dynamics of magnetic vortices, Nuclear Phys. B, 360 (1991), pp. 425-462.

[RT] J. ROBERT AND C. ThOMPSON, Dynamics of the classical Heisenberg spin chain, J. Phys. A, 21 (1988), pp. 1769-1780.

[S] J. SнатAн, Weak solutions and development of singularities in the $S U(2) \sigma-m o d e l$, Comm. Math. Phys., 41 (1988), pp. 459-469.

[SS] J. Shatah AND M. StRUWE, Regularity results for nonlinear wave equations, Ann. Math., 138 (1993), pp. 503-518.

[T] L. A. TAKHTAJAN, Integration of the continuous Heisenberg spin chain through the inverse scattering method, Phys. Lett. A, 64 (1977), pp. 235-237.

[W] Y. D. WANG, Ferromagnetic chain equation from a closed Riemannian manifold into $S^{2}$, International J. Math., 6:1 (1995), pp. 93-102.

[W1] Y. D. WANG, Heisenberg chain systems from compact manifolds into $S^{2}$, J. Math. Phys., 39:1 (1998), pp. 363-371.

[Z] Y. ZHOU, Global weak solutions for $1+2$ dimensional wave maps into homogeneous spaces, Ann. Inst. H. Poincaré Anal. Nonlinéaire, 16 (1999), pp. 411-422. 
[ZG] Y. ZHOU AND B. GuO, Weak solution of system of ferromagnetic chain with several variables, Science in China A, 27 (1987), pp. 1251-1266.

[ZGT] Y. ZHOU, B. GuO, AND S. TAN, Existence and uniqueness of smooth solution for system of ferromagnetic chain, Science in China A, 34 (1991), pp. 257-266.

[ZS] V. E. ZAKHAROV AND A. B. ShaBat, Exact theory of two-dimensional self-focusing and one-dimensional self-modulation of waves in nonlinear media, J. Experim. Theore. Phys., 34 (1972), pp. 62-69.

[ZT] V. E. ZAKHAROV AND L. A. TAKHTAJAX, Equivalence of a nonlinear Schroödinger equation and a Heisenberg ferromagnet equation, Theore. Math. Phys., 38 (1979), pp. 17-23. 
